\title{
Boltzmann-Machine Learning of Prior Distributions of Binarized Natural Images
}

\author{
${ }^{1,2}$ Tomoyuki Obuchi, ${ }^{2}$ Hirokazu Koma, and ${ }^{3}$ Muneki Yasuda \\ ${ }^{1}$ Department of Computational Intelligence and Systems Science, Tokyo Institute of Technology, \\ Yokohama, 226-8502, Japan. \\ ${ }^{2}$ Cybermedia Center, Osaka University, Osaka 560-0043, Japan. \\ ${ }^{3}$ Graduate School of Science and Engineering, Yamagata University, Yonezawa, 992-8510, Japan.
}

Prior distributions of binarized natural images are learned by using a Boltzmann machine. According the results of this study, there emerges a structure with two sublattices in the interactions, and the nearest-neighbor and next-nearest-neighbor interactions correspondingly take two discriminative values, which reflects the individual characteristics of the three sets of pictures that we process. Meanwhile, in a longer spatial scale, a longer-range, although still rapidly decaying, ferromagnetic interaction commonly appears in all cases. The characteristic length scale of the interactions is universally up to approximately four lattice spacings $\xi \approx 4$. These results are derived by using the mean-field method, which effectively reduces the computational time required in a Boltzmann machine. An improved mean-field method called the Bethe approximation also gives the same results, as well as the Monte Carlo method does for small size images. These reinforce the validity of our analysis and findings. Relations to criticality, frustration, and simple-cell receptive fields are also discussed.

KEYWORDS: Boltzmann machine, Markov random fields, neural networks, image processing, mean-field methods

\section{Introduction}

The Bayesian framework of image processing was initiated in, ${ }^{1)}$ and is currently an active research field in several disciplines. ${ }^{2-7)}$ This research field has advanced in close relation to neural networks, ${ }^{5)}$ the interest in which has recently begun to grow rapidly by virtue of new algorithms and concepts, such as multiple layers of hidden units allowing high orders of statistics to be taken into account naturally, ${ }^{8-10)}$ as well as the sparseness of signals allowing input signals to be expressed efficiently and universally. ${ }^{4}$ ) These advances have provided increasingly improved generative models of images that need to be processed.

The Bayesian image processing framework inevitably requires to introduce prior distributions of the images. Most earlier studies, in a sense, focused on using one prior distribution that is applicable 
to any image of interest. However, we may use different prior distributions according to the sets of images we are processing, which may result in a better image processing performance.

To examine this possibility, in this study, we evaluate the prior distributions of several different sets of binarized natural images by using a Boltzmann machine. ${ }^{11)}$ The Boltzmann machine is a classical model of neural networks and is much simpler than recently developed models, such as deep belief networks, which consist of multiple layers of hidden units, ${ }^{8-10)}$ or methods that utilize sparseness. ${ }^{4)}$ However, to the best of our knowledge, even such a simple model has never been examined in this context. We considered that the simple Boltzmann machine would be a good starting point for the present purpose, because the correlations among visible units of the Boltzmann machine become simpler than those of the advanced models with hidden units, and thus, it should be easier to find individual characteristics in different categories of natural images.

In particular, the fully connected Boltzmann machine is treated in the framework of maximum likelihood estimation. Maximization of the likelihood is known to be difficult in general, and hence, we use two variants of the mean-field approximations in this study: the naive mean-field approximation and the improved one, called Bethe approximation. The consistency between these two variants supports the validity of our approximation. In the analysis, we treat three sets of images as representatives: aerial geographic pictures, face pictures, and forest pictures. These pictures have discriminable properties, such as domain and fractal structures, and we can expect that some discriminative characteristics would appear in the learning results.

Our learning results of the Boltzmann machine in fact show that certain discriminative characteristics exist in different individual sets of images, which clearly appear in the interactions of the Boltzmann machine. One strong characteristic is the value of the nearest neighboring (NN) interactions. These can strongly change according to the individual sets of images, and can be both positive and negative. The next-nearest-neighboring (NNN) interactions also depend on the individual sets, but the tendency is relatively weak. Meanwhile, we also found some properties that the different sets of images have in common. One is the length scale of the interactions. We observed that, for a spatial range longer than the next-nearest neighbors, ferromagnetic interactions commonly appear and they decay in a rapid (approximately exponential) manner; the characteristic length scale of interactions is commonly approximately $\xi \approx 4$. This universality of rapidly decaying interactions may explain the good performance in image segmentation reported in, ${ }^{12)}$ where an exponentially decaying interaction in the Markov random field was introduced. In addition, the Boltzmann machine after learning universally shows an absence of criticality. This may be a surprising observation, because natural images are known to exhibit a certain criticality (power law of the Fourier amplitude in the spatial frequency). ${ }^{2,3,5,7,13)}$ This absence of criticality in the Boltzmann machine after learning implies that the 
criticality in natural images originates in higher order statistics than the second one, since the Boltzmann machine takes into account statistics only up to the second order. In addition, it is commonly observed that frustration is absent or quite weak, even when it exists. One possible consequence of the frustration being very weak is a smooth phase space with a few minima. This potentially provides an intuitive interpretation of the energy and minima, as suggested in. ${ }^{13)}$

The remaining parts of this paper are organized as follows. In the following section, we introduce the Boltzmann machine and state the setup of our problem. The Boltzmann machine is known to be computationally infeasible and thus we use two approximations, which are also explained in the section. In sec. 3, we display the estimated parameters of the Boltzmann machine. The results described above are illustrated in detail using these numerical data. The robustness of the result is also examined in this section by Monte Carlo simulations for small sizes and by testing a number of dithering methods. In sec. 4, we discuss the criticality and a relation to simple-cell receptive fields, and finally, propose a model of the prior distribution of natural images based on our observations. The final section is devoted to the conclusion.

\section{Model, Task and Methods}

\subsection{Boltzmann Machine}

In this section, we explain the definition of the Boltzmann machine to state our objectives. The Boltzmann machine is defined by the following energy function or the Hamiltonian

$$
\mathcal{H}(\boldsymbol{S} \mid \boldsymbol{w}, \boldsymbol{h})=-\sum_{\langle i, j\rangle} w_{i j} S_{i} S_{j}-\sum_{i} h_{i} S_{i},
$$

where $S_{i}= \pm 1$ denotes the bit or spin, $w_{i j}$ the interaction, and $h_{i}$ the field. The probability distribution of spins is given by

$$
p(\boldsymbol{S} \mid \boldsymbol{w}, \boldsymbol{h})=\frac{1}{Z(\boldsymbol{w}, \boldsymbol{h})} e^{-\mathcal{H}(\boldsymbol{S} \mid \boldsymbol{w}, \boldsymbol{h})} .
$$

Note that our Boltzmann machine is fully connected and there is no hidden unit.

The average of an observable $\hat{O}$ over this distribution is hereafter denoted by

$$
\sum_{S} \hat{O} p(\boldsymbol{S} \mid \boldsymbol{w}, \boldsymbol{h}) \equiv\langle\hat{O}\rangle_{w, \boldsymbol{h}}
$$

\subsection{Learning of Effective Interactions in Images}

Let us consider the Boltzmann machine in image processing. Suppose we have $B$ pictures $\left\{\boldsymbol{S}^{(\mu)}\right\}_{\mu=1, \cdots, B}$ and assume that they are generated from a certain distribution. This distribution is ap- 
proximated by the empirical distribution

$$
p_{D}\left(S \mid\left\{\boldsymbol{S}^{(\mu)}\right\}\right)=\frac{1}{B} \sum_{\mu=1}^{B} \delta_{S, S^{(\mu)}}
$$

where $\delta_{S, T}$ denotes the indicator function giving unity if $\boldsymbol{S}=\boldsymbol{T}$, and zero otherwise. We write the average by this distribution as $\sum_{S} p_{D} \hat{O}=\langle\hat{O}\rangle_{D}$. A typical learning scheme is formulated to reproduce the average of several observables over the empirical distribution by using that over the Boltzmann distribution

$$
\langle\hat{O}\rangle_{w, h}=\langle\hat{O}\rangle_{D}
$$

We seek $\boldsymbol{w}, \boldsymbol{h}$ to satisfy this moment-matching condition for appropriate observables. The choice of observables clearly influences the results; here we choose the first and second moments of spins, magnetization and pairwise correlations, respectively. Let us fix the notations of the relevant quantities

$$
\begin{aligned}
& m_{i}=\left\langle S_{i}\right\rangle_{w, \boldsymbol{h}}, \mu_{i}=\left\langle S_{i}\right\rangle_{D}, \\
& C_{i j}=\left\langle S_{i} S_{j}\right\rangle_{w, \boldsymbol{h}}-m_{i} m_{j}, \quad \Gamma_{i j}=\left\langle S_{i} S_{j}\right\rangle_{D}-\mu_{i} \mu_{j} .
\end{aligned}
$$

This choice of observables is natural, since they are in a conjugate relation with $\boldsymbol{h}$ and $\boldsymbol{w}$. In particular, the solution of these equations, $p\left(\boldsymbol{S} \mid \boldsymbol{w}^{*}, \boldsymbol{h}^{*}\right)$, can be written as the maximizer of the log likelihood

$$
p\left(\boldsymbol{S} \mid \boldsymbol{w}^{*}, \boldsymbol{h}^{*}\right)=\arg \max _{p(\boldsymbol{S} \mid \boldsymbol{w}, \boldsymbol{h})}\left\{\sum_{S} p_{D}\left(\boldsymbol{S} \mid\left\{\boldsymbol{S}^{(\mu)}\right\}\right) \ln p(\boldsymbol{S} \mid \boldsymbol{w}, \boldsymbol{h})\right\} .
$$

From this equation, the moment-matching conditions with respect to magnetizations and pair-wise correlations are naturally derived. The task to be solved in this study is to infer $\boldsymbol{w}$ and $\boldsymbol{h}$ from several sets of natural images and to find characteristic features.

\subsection{Mean-Field Methods}

To find the optimal values of $\boldsymbol{w}$ and $\boldsymbol{h}$, steepest-descent-type algorithms with respect to $\boldsymbol{w}$ and $\boldsymbol{h}$ are typically used. However, at each step of changing $\boldsymbol{w}$ and $\boldsymbol{h},\langle\hat{O}\rangle_{\boldsymbol{w}, \boldsymbol{h}}$ need to be evaluated, which is infeasible for large systems, since the evaluation of the partition function $Z(\boldsymbol{w}, \boldsymbol{h})$ in general requires an exponentially growing time as the system size increases. To overcome this difficulty, we employ two variants of the mean-field methods. Namely, the first is the naive mean-field method (NMF) ${ }^{14-16)}$ and the second is the improved mean-field method, called Bethe approximation (BA). ${ }^{17,18)}$

The original model of the Boltzmann machine (2) involves interactions among variables, and thus, it is difficult to calculate the partition function. To overcome this difficulty, the basic strategy of the mean-field methods is to decompose this multi-body probability distribution into a batch of effective probability distributions consisting of a small number of variables. The NMF breaks eq. (2) into a 
batch of single-spin distributions, while the BA approximates eq. (2) by an appropriate combination of single-spin and two-spin distributions. Here, we omit the detailed descriptions and present only the results. Readers interested in the details may refer to. ${ }^{14-20)}$

The NMF results are

$$
\begin{gathered}
w_{i j}=-\left(\Gamma^{-1}\right)_{i j}+\frac{\delta_{i j}}{1-\mu_{i}^{2}}, \\
h_{i}=\tanh ^{-1} \mu_{i}-\sum_{j} w_{i j} \mu_{j} .
\end{gathered}
$$

Note that, in the original model (2), the self-interaction terms $w_{i i}$ have no meaning, but in the inverse problem these terms should match the dimension of the given data $\boldsymbol{\mu}$ and $\boldsymbol{\Gamma}$. In addition, the BA provides the formulas

$$
\begin{aligned}
& w_{i j}=\tanh ^{-1}\left\{\mu_{i} \mu_{j}-\frac{1}{2\left(\Gamma^{-1}\right)_{i j}} D_{i j}\right. \\
& \left.+\frac{1}{\left(\Gamma^{-1}\right)_{i j}} \sqrt{\frac{1}{4}-\mu_{i} \mu_{j}\left(\Gamma^{-1}\right)_{i j} D_{i j}+\left(2 \mu_{i}^{2} \mu_{j}^{2}-\mu_{i}^{2}-\mu_{j}^{2}\right)\left(\Gamma^{-1}\right)_{i j}^{2}}\right\} \\
& h_{i}=\tanh ^{-1}\left(\mu_{i}\right)-\sum_{j} \tanh ^{-1}\left(t_{i j} f\left(\mu_{j}, \mu_{i}, t_{i j}\right)\right),
\end{aligned}
$$

where $t_{i j}=\tanh w_{i j}$ and

$$
\begin{aligned}
& D_{i j}=\sqrt{1+4\left(1-\mu_{i}^{2}\right)\left(1-\mu_{j}^{2}\right)\left(\Gamma^{-1}\right)_{i j}^{2}}, \\
& f\left(\mu_{1}, \mu_{2}, t\right)=\frac{1-t^{2}-\sqrt{\left(1-t^{2}\right)^{2}-4 t\left(\mu_{1}-\mu_{2} t\right)\left(\mu_{2}-\mu_{1} t\right)}}{2 t\left(\mu_{2}-\mu_{1} t\right)} .
\end{aligned}
$$

The BA can be expected to yield more accurate results, since it includes higher body correlations than does the NMF, and the computational time of eqs. $(11,12)$ remains comparable with that of NMF $(9,10)$. The above formula of the BA is taken from. ${ }^{18)}$

\section{Results}

In this section, we show the estimated $\boldsymbol{w}$ and $\boldsymbol{h}$ for three sets of natural pictures. One comprises aerial pictures, the second human face pictures, and the third forest pictures. We selected these three sets rather arbitrarily, meaning that each set appears to have some discriminative properties, such as the sizes of clusters and shapes of edges, of the other two sets.

Each set of pictures, aerial, face, and forest, are downloaded from the database of the Geospatial Information Authority of Japan, ${ }^{21)}$ the Color FERET Database, ${ }^{22)}$ and the SUN Database, ${ }^{23)}$ respectively. The original pictures are multi-colored and are binarized by ImageMagick, ${ }^{24)}$ and we assume a black dot is represented by +1 and a white one by -1 . More precisely, a spatial color quantization ${ }^{25)}$ with gray colorspace and the Riemersma dither ${ }^{26)}$ are employed in the binarizing process. Typical ex- 
amples of each set, aerial, face, and forest pictures, are given in Figs. 1-3, respectively. The empirical distribution of pictures having a fixed size is generated by cutting many binarized pictures into several square-shaped patches with equal sides having a length of $L$, and mixing them equally.
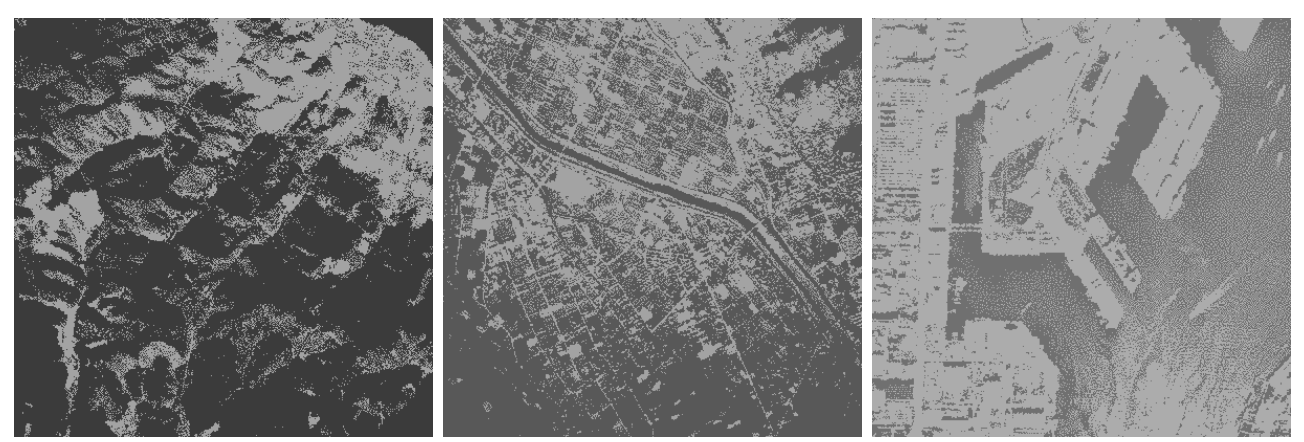

Fig. 1. Typical binarized aerial pictures used in learning. From. ${ }^{21)}$
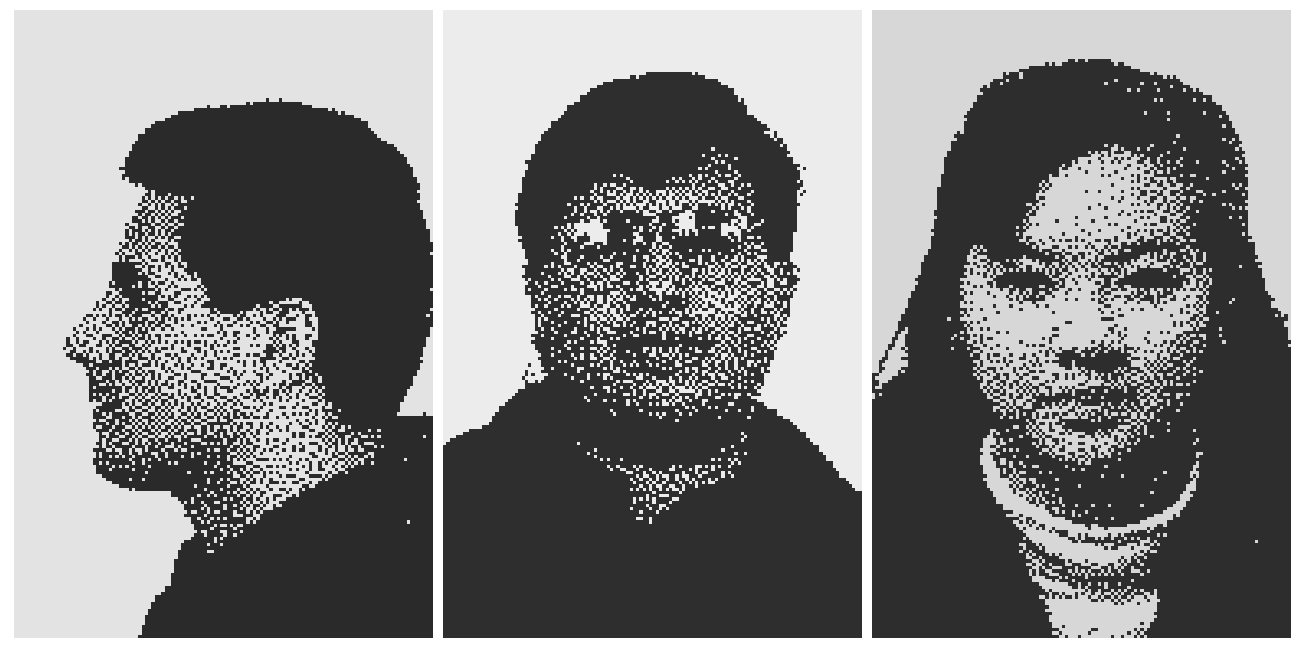

Fig. 2. Typical binarized face pictures used in learning. From. ${ }^{22)}$

For simplicity of notation, hereafter, let us represent each site $i$ by the orthogonal coordinates $\boldsymbol{r}_{i}=\left(x_{i}, y_{i}\right)$ with integers $x_{i}, y_{i}=1,2, \cdots$. We allocate these coordinates $\left(x_{i}, y_{i}\right)$ to pixels, as shown in Fig. 4. The interaction between pixels $i$ and $j$ is rewritten as $w_{i j}=w\left(\boldsymbol{r}_{i}, \boldsymbol{r}_{j}\right)$. We assume the distance between two pixels $i$ and $j$ is defined in the Euclid manner, $r_{i j}=\sqrt{\left(x_{i}-x_{j}\right)^{2}+\left(y_{i}-y_{j}\right)^{2}}$. 

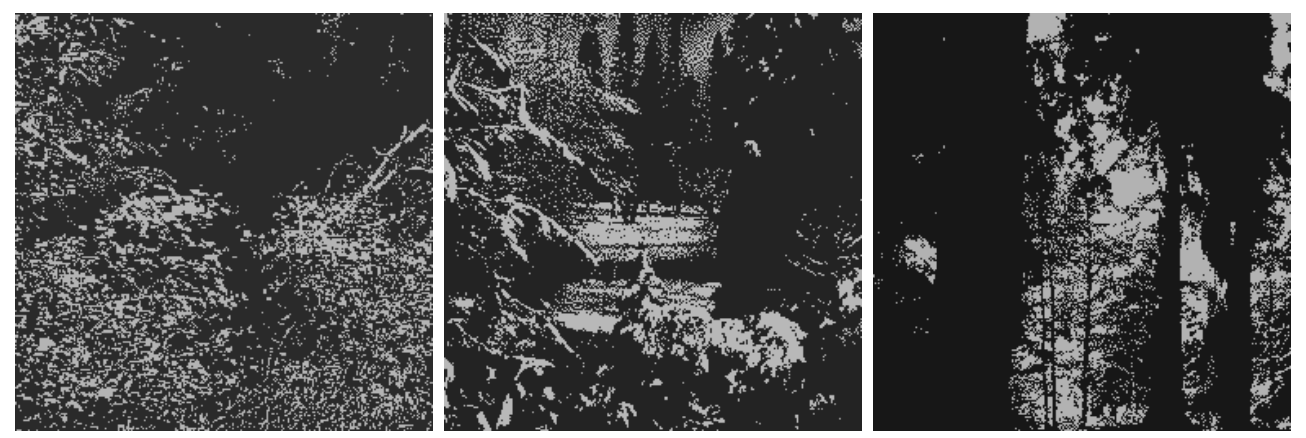

Fig. 3. Typical binarized forest pictures used in learning. From. ${ }^{23)}$

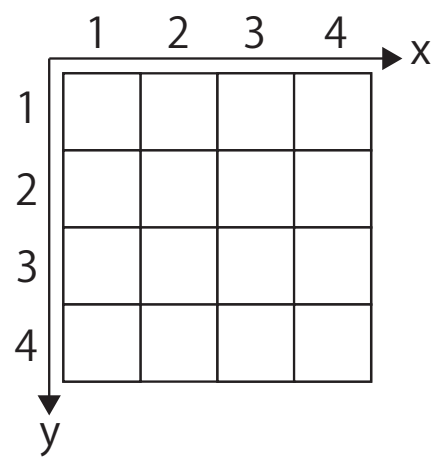

Fig. 4. Correspondence between coordinates and pixels. Each square represents a pixel. The origin locates at the upper left edge of the patch.

\subsection{Inferred Interactions}

\subsubsection{Aerial Pictures}

In this section, we observe the inferred interactions of the aerial pictures. As clarified later by a comparison of this set with the other two sets of pictures, this case is the simplest, where the interactions take only positive values.

First, let us observe some basic behavior of $w\left(\boldsymbol{r}_{i}, \boldsymbol{r}_{j}\right)$ inferred by the NMF in eqs. $(9,10)$, focusing in particular on the dependency on the distance $r=\left|\boldsymbol{r}_{j}-\boldsymbol{r}_{i}\right|$ and on the origin $\boldsymbol{r}_{i}$. In this context, we rewrite $w\left(\boldsymbol{r}_{i}, \boldsymbol{r}_{j}\right)=w\left(r \mid \boldsymbol{r}_{i}\right)$. In Fig. 5, we plot $w\left(r \mid \boldsymbol{r}_{i}\right)$ of common $y$-coordinate $y_{i}=y_{j}$ against $r=\left|x_{j}-x_{i}\right|$ with $x_{j}=x_{i}+1, x_{i}+2, \cdots x_{i}+8$. Namely, we plot the interactions in the row as they change across the column from left to right. Several different curves are shown when the origin $\boldsymbol{r}_{i}=\left(x_{i}, y_{i}\right)$ changes. The size and number of the patches are $L=16$ and $B=100,000$, respectively. We confirm that $w\left(r \mid \boldsymbol{r}_{i}\right)$ along the opposite direction, namely, from right to left, shows quite similar behavior, and that along the vertical direction in the column it does as well. Thus, Fig. 5 is a good representative. Crucial observations from Fig. 5 are as follows. 

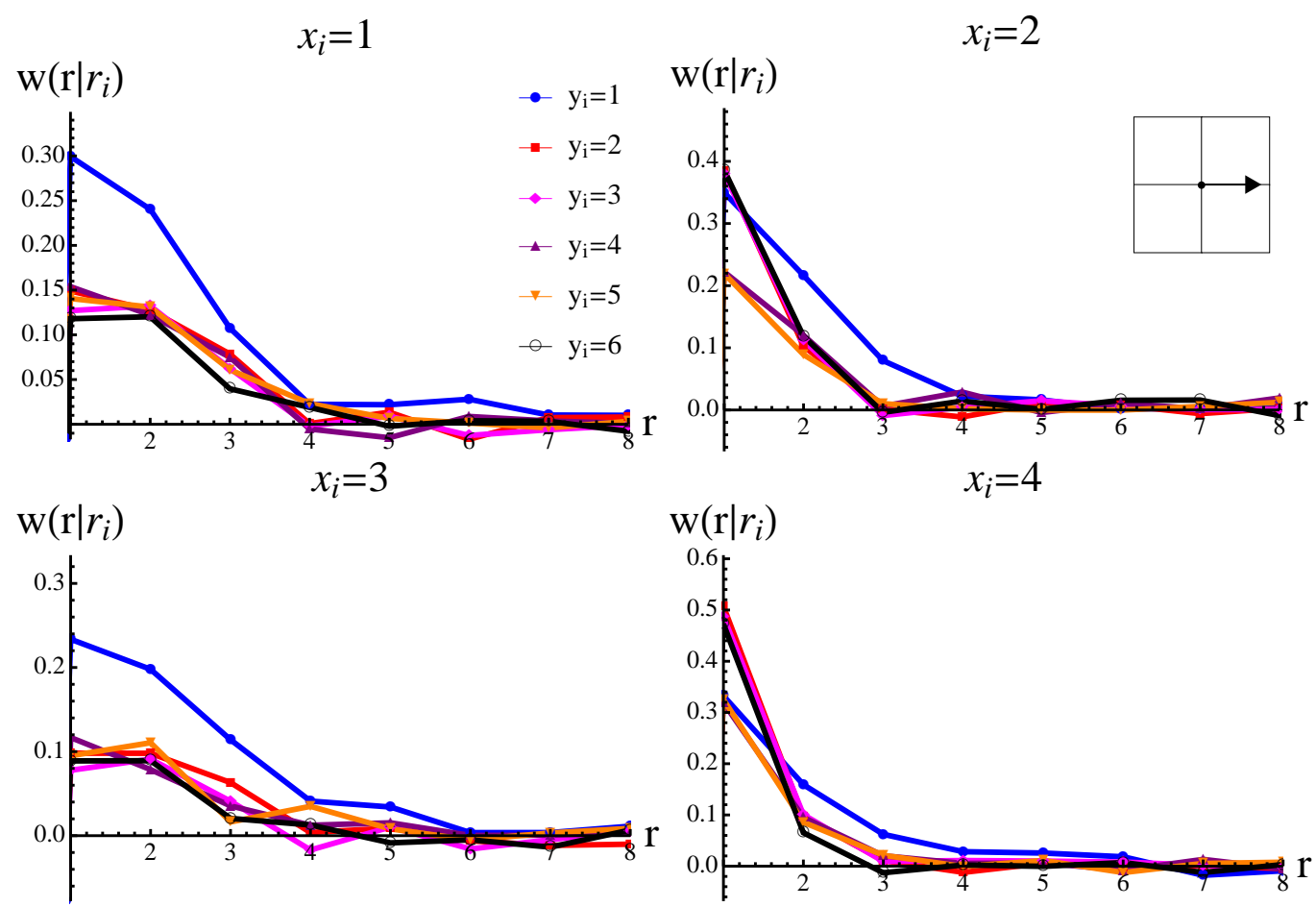

Fig. 5. (Color online) Plots of interactions $w\left(r \mid \boldsymbol{r}_{i}\right)$ against distance $r=\left|x_{j}-x_{i}\right|$ in a common row $y_{i}=y_{j}$ as the origin of the plot $\boldsymbol{r}_{i}=\left(x_{i}, y_{i}\right)$ changes (the top-right inset represents the moving direction of $\left.\boldsymbol{r}_{j}\right)$. The interactions are inferred by the NMF using $B=100,000$ patches of size $L=16$ created from the aerial pictures.

- Most of the interactions are positive for $r \leq 4 \equiv \xi$ and almost vanish for $r>\xi$.

- A clear boundary effect exists for $y_{i}=1$ (blue curves). Namely, the absolute values of $w\left(r \mid y_{i}=1\right)$ are larger than the other values of $y_{i}$.

- A periodic behavior exists in the interactions, in particular for the NN ones at $r=1$. Namely, the absolute values of the NN interactions for $x_{i}=2$ and 4 are similar, as well as those for $x_{i}=1$ and 3 , but the former for $x_{i}=2$ and 4 are larger than the latter for $x_{i}=1$ and 3 .

- A weaker periodic behavior appears to exist among different $y_{i}$ for even $x_{i}=2$ and 4 . For example, for $x_{i}=2$, curves with $y_{i}=2,3$ and 6 behave similarly, as do other curves of $y_{i}=4$ and 5 .

The first three characteristics listed above are clearer than the fourth, and we checked that they are common among other rows and columns and similar counterparts exist for the other sets of pictures, faces and forests. Hence, the analyses below are based on these three findings. The final fourth characteristic, a weaker periodic behavior, also seems to hold for the other two sets of pictures, but we avoid performing the analysis since it is not easy to treat systematically because of the weakness of the tendency and the complexity of the periodicity.

Next, we observe the orientation dependency of $w\left(\boldsymbol{r}_{i}, \boldsymbol{r}_{j}\right)$. We plot $w\left(\boldsymbol{r}_{i}, \boldsymbol{r}_{j}\right)$ when we vary $\boldsymbol{r}_{j}$ as $\boldsymbol{r}_{j}=\left(x_{i}+s, y_{i}+s\right)$ with $s=1,2, \cdots, 6$, namely, along the downward slope of the 45-degree angle 
from fixed $\boldsymbol{r}_{i}$. The distance then becomes $r_{i j}=\sqrt{2} s$. Fig. 6 again shows that the interactions are
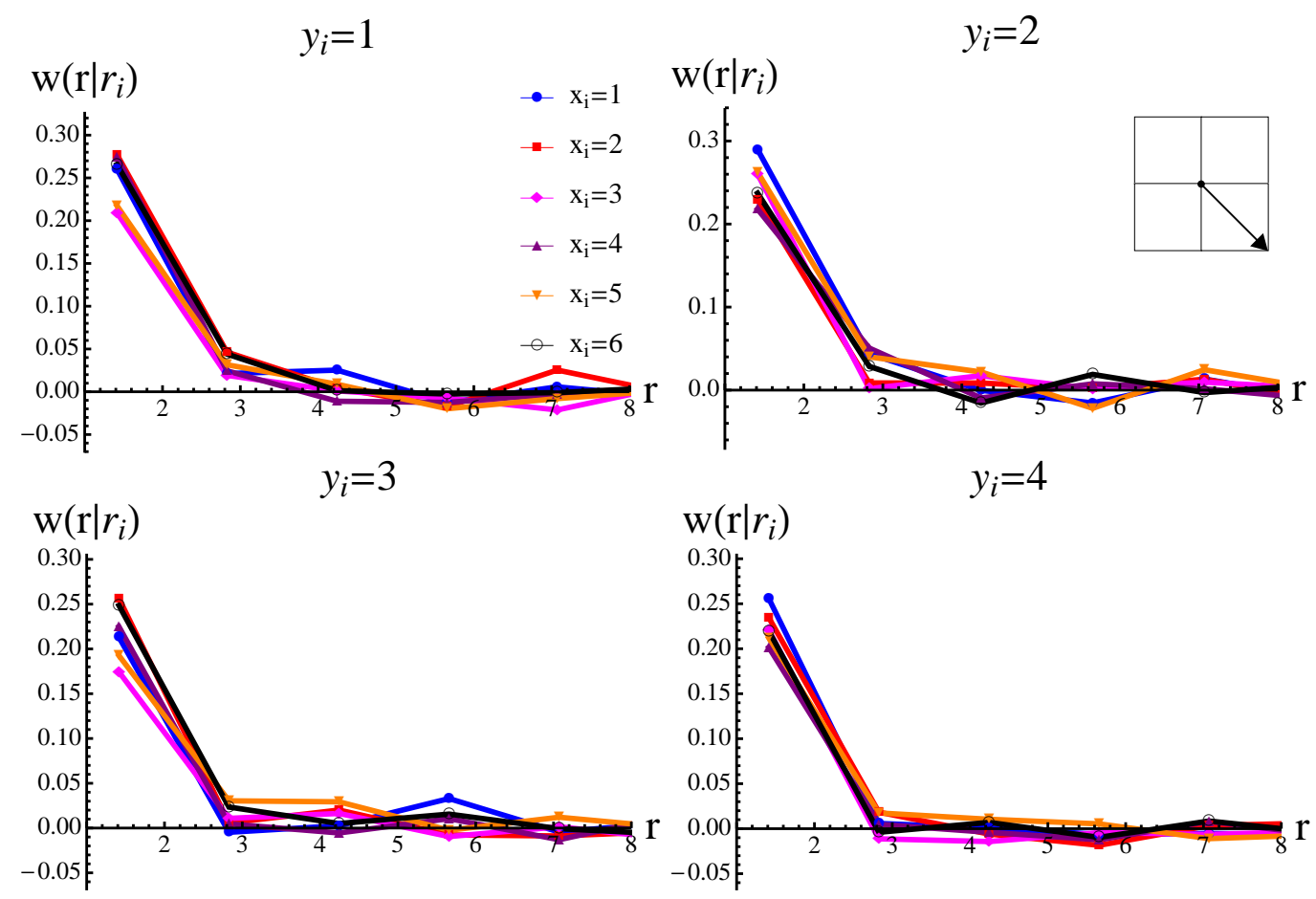

Fig. 6. (Color online) Plots of interactions $w\left(r \mid \boldsymbol{r}_{i}\right)$ against distance $r_{i j}=\sqrt{2} s$ with $s=1,2, \cdots 6$ along the downward slope of the 45-degree angle from fixed $\boldsymbol{r}_{i}$. The same images and parameters as in Fig. 5 are used.

ferromagnetic and are not long ranged; they almost vanish for $r \geq 3$. This length scale is shorter than that shown in Fig. 5, implying that the interactions along the horizontal or vertical lines are stronger than those along other inclined directions. Here, it is not easy to find any clear dependency on the choice of origins $\left\{\boldsymbol{r}_{i}\right\}$, and thus, the periodicity becomes weaker in this direction. The absence of direction dependency is again checked by examining the interactions along the opposite direction and those along the upward slope of the 45-degree angle.

The above findings of periodicity imply the existence of a sublattice structure as represented in the left panel of Fig. 7. For convenience later in this paper, we also depict the sublattice structure of the NNN interactions, which is not seen in aerial pictures, but is seen in face pictures, as shown in the center panel in Fig. 7. This sublattice structure periodically paves the whole patch without overlap, and thus each link is uniquely determined to have which color. Note that the color just indicates which sublattice the link belongs to, and has no relation to the sign of the corresponding interaction. The sublattice structure of the $\mathrm{NN}$ interactions is captured well by the histogram of the $\mathrm{NN}$ interactions $P\left(w_{i j} \mid r_{i j}=1\right)$, which is shown in the right panel of Fig. 7. The histograms of these two different types of interaction (blue and magenta) are clearly differently distributed. This evidence strongly supports 

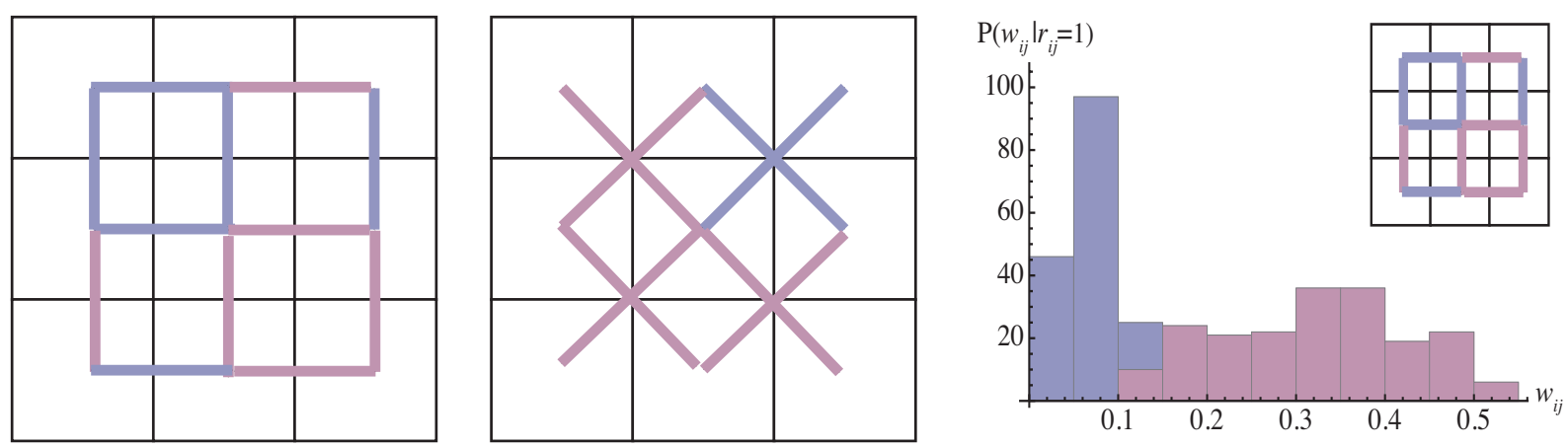

Fig. 7. (Color online) (Left) Sublattice structure implied by the periodicity in the NN interactions, where the squares correspond to pixels and links between neighboring squares represent the interactions. Two types of link, which are represented by blue and magenta bars, emerge. (Center) Sublattice structure in the NNN interactions (clearly seen in the case of face pictures). (Right) Histogram of NN interactions discriminating the two types of links, which are represented by two different colors, blue and magenta, in correspondence with the left panel. The inset is the reduced scale version of the left panel. The distribution of the values of $w_{i j}$ are clearly different in these two types of NN links.

the presence of the sublattice structure.

The system-size dependence of the above results, in particular the characteristic length scale of the interaction range, needs to be examined. For this purpose, we define the following averaged interactions

$$
\begin{gathered}
\bar{w}\left(r \mid\left(\hat{x}_{i}, \hat{y}_{i}\right)\right)=\frac{1}{2(L-2)}\left\{\sum_{x_{j}=x_{i}+1}^{L} \sum_{y_{i}=2}^{L-1} w\left(r_{i j}=\left|x_{j}-\hat{x}_{i}\right| \mid \boldsymbol{r}_{i}=\left(\hat{x}_{i}, y_{i}\right)\right) \delta\left(r-r_{i j}\right)\right. \\
\left.+\sum_{y_{j}=y_{i}+1}^{L} \sum_{x_{i}=2}^{L-1} w\left(r_{i j}=\left|y_{j}-\hat{y}_{i}\right| \mid \boldsymbol{r}_{i}=\left(x_{i}, \hat{y}_{i}\right)\right) \delta\left(r-r_{i j}\right)\right\} .
\end{gathered}
$$

As seen thus far, the behavior of $w\left(r \mid \boldsymbol{r}_{i}\right)$ in $r \geq 2$ is stable and does not significantly fluctuate over different directions and origins, which justifies taking this average. The first sum in eq. (15) is the contributions of $w\left(r \mid \boldsymbol{r}_{i}\right)$ along the horizontal direction moving right from $\hat{x}_{i}$ and the second is those along the vertical direction moving down from $\hat{y}_{i}$. Contributions from the boundaries (blue curves in Fig. 5) are excluded in eq. (15). We can see sublattices in Fig. 7 by appropriately choosing the coordinates $\left(\hat{x}_{i}, \hat{y}_{i}\right)$. We define and plot $\overline{w_{A}}(r) \equiv \bar{w}(r \mid(1,1))$ and $\overline{w_{B}}(r) \equiv \bar{w}(r \mid(2,2))$ of different patch sizes $L=8,16$, and 32 in Fig. 8. The number of used patches in the plots shown in Fig. 8 is $B=$ 400, 000 and 23400 for $L=8$ and 32, respectively. The error bar of each data point in Fig. 8 is defined by $\sigma(r) / \sqrt{2(L-2)}$, where $\sigma(r)$ is the standard deviation of the terms in eq. (15) from $\bar{w}\left(r \mid\left(\hat{x}_{i}, \hat{y}_{i}\right)\right)$. The results clearly demonstrate the absence of the size effect, which is consistent with the characteristic length scale of $w\left(r \mid \boldsymbol{r}_{i}\right)$ being $\xi \approx 4$. Patches larger than $L=8$, which is twice $\xi \approx 4$, suffice to reproduce our findings. 

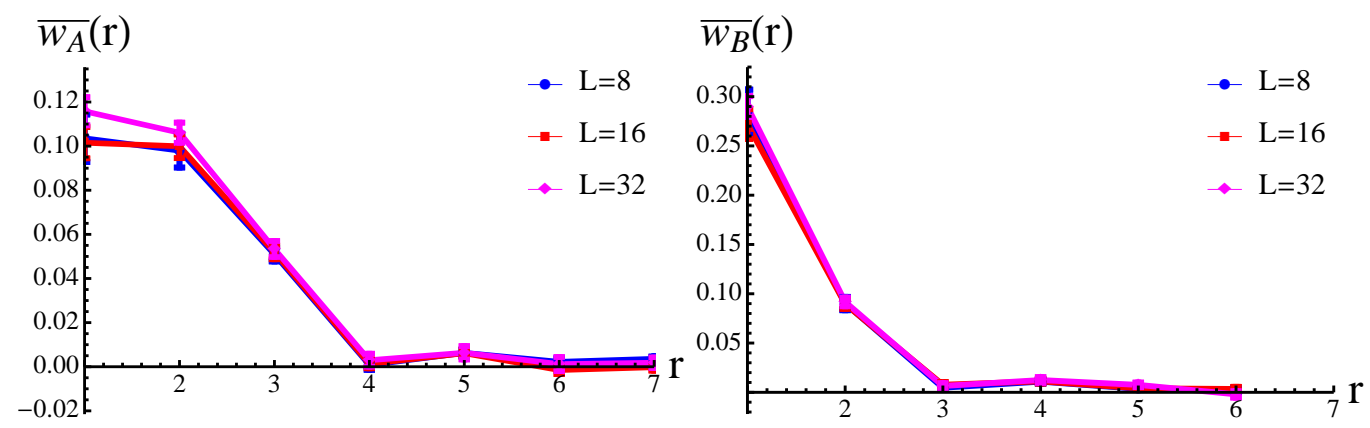

Fig. 8. (Color online) Plots of $\overline{w_{A}}(r) \equiv \bar{w}(r \mid(1,1))$ (left) and $\overline{w_{B}}(r) \equiv \bar{w}(r \mid(2,2))$ (right) for different patch sizes $L=$ 8, 16, and 32. The number of used patches is $B=400,000$ and 23400 for $L=8$ and 32, respectively. The size effect is clearly absent.

Next, we compare the results obtained by the NMF and BA shown in Fig. 9. We can see the
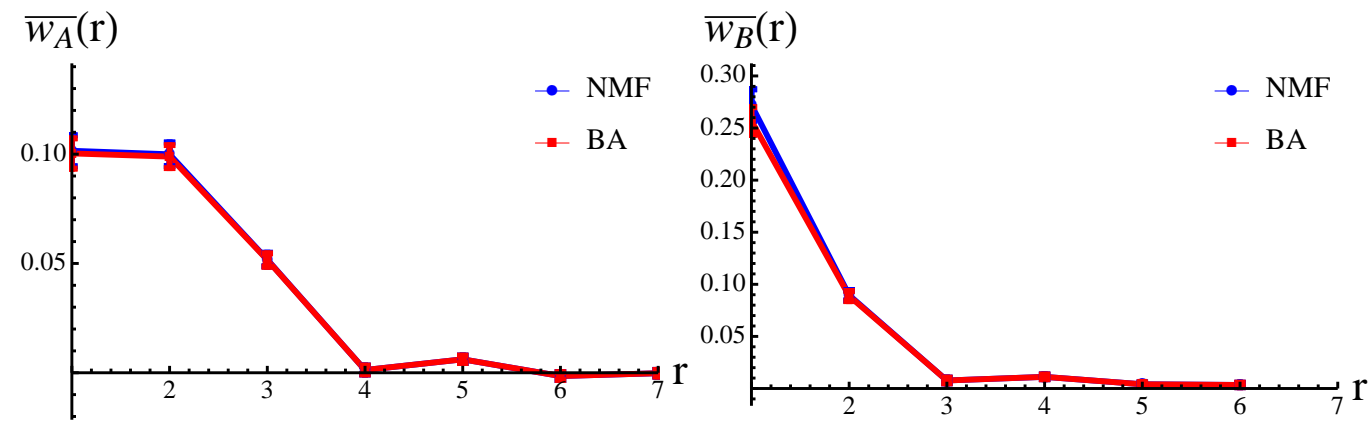

Fig. 9. (Color online) Plots of $\overline{w_{A}}(r) \equiv \bar{w}(r \mid(1,1))$ (left) and $\overline{w_{B}}(r) \equiv \bar{w}(r \mid(2,2))$ (right) inferred by the NMF and BA for patch size $L=16$. The absolute values of $w$ are slightly larger in the NMF than in the BA, but the difference is negligibly tiny.

difference between the NMF and the BA is negligibly small. Thus, for our purpose of finding the characteristics of the interactions, the NMF appears to suffice, at least for the aerial pictures we treat.

\subsubsection{Face Pictures}

Next, we show the learned interactions of face pictures. In general, we can identify three regions in each picture in this category: the background, the hair and cloth, and the face itself, as seen in Fig. 2. The face region is expressed by patterns where both black and white pixels emerge frequently and alternately, which is considered to be produced by a dither process to discriminate this region from the others. Presumably as a result of this dither process, some antiferromagnetic interactions, which are absent in the aerial pictures, are observed, as seen below.

We first observe the NMF results in the case of the aerial pictures. In Fig. 10, we display the 
interactions $w\left(r \mid \boldsymbol{r}_{i}\right)$ against the distance $r$ in a column, which correspond to Fig. 5. Again, we see that

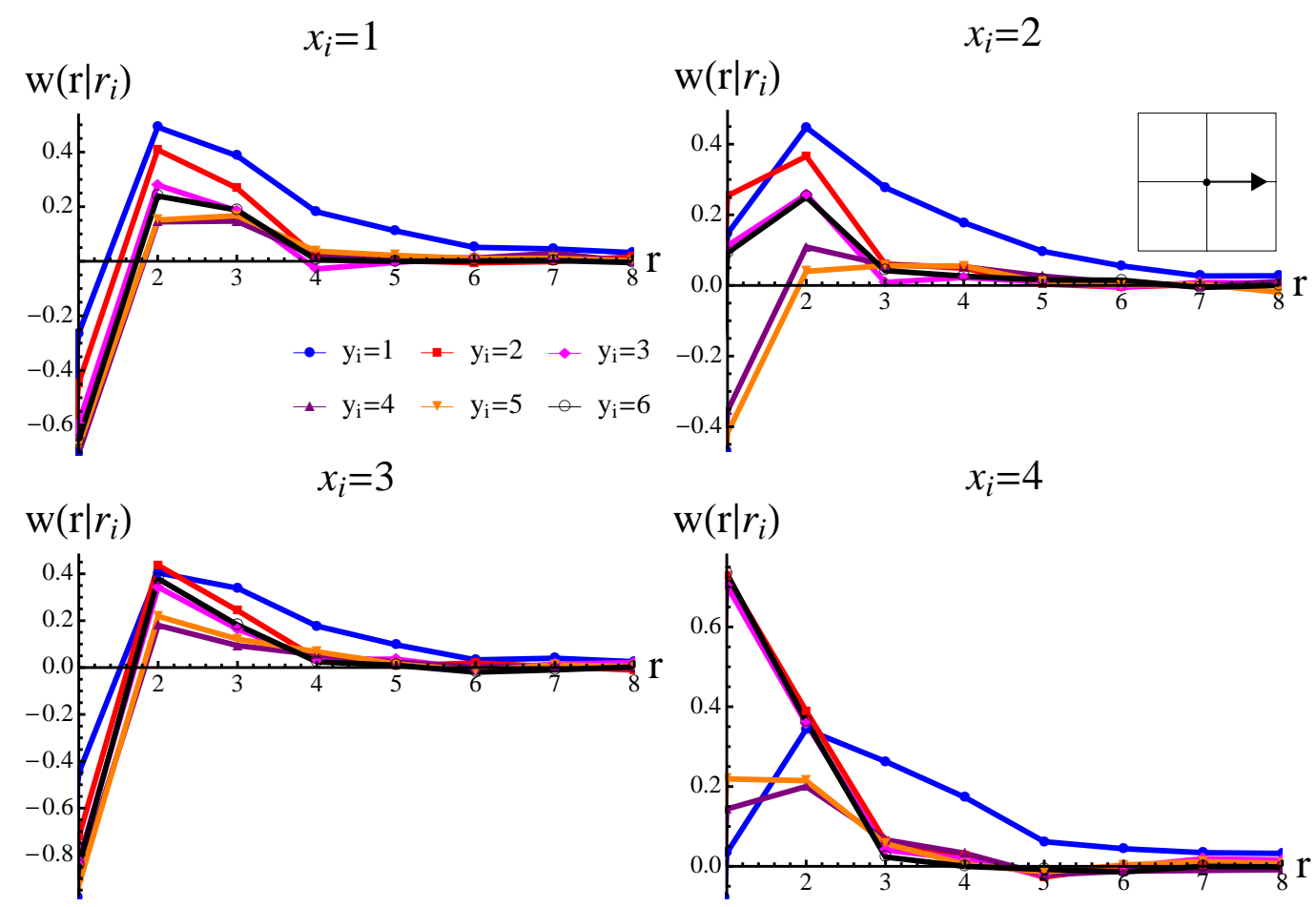

Fig. 10. (Color online) Plots of interactions $w\left(r \mid \boldsymbol{r}_{i}\right)$ against distance $r=\left|x_{j}-x_{i}\right|$ in a common row $y_{i}=y_{j}$ as the origin of the plot $\boldsymbol{r}_{i}=\left(x_{i}, y_{i}\right)$ changes (the top-right inset represents the moving direction of $\left.\boldsymbol{r}_{j}\right)$. The interactions are inferred by the NMF using $B=576,096$ patches of size $L=16$ created from face pictures.

the inferred interactions decrease around $r \approx 4$. The boundary effect at $y_{i}=1$ is also present and the NN interactions appear to have a periodicity similar to that seen in Fig. 7. However, a new observation in Fig. 10 is

- Some NN interactions take negative values.

As noted above, these antiferromagnetic interactions are considered to emerge for expressing patterns where black and white pixels alternatively appear, which are presumably produced by a dither process to discriminate the face from other regions.

The orientation dependency of the inferred interactions is examined by observing $w\left(r \mid \boldsymbol{r}_{i}\right)$ along the downward slope as in Fig. 6. The results are shown in Fig. 11. New observations are:

- Some NNN interactions also take negative values.

- A periodicity in the NNN interactions is present. Namely, the behavior of the NNN interactions at $y_{i}=1$ and 3 is similar, while it differs from those at $y_{i}=2$ and 4 with similar values.

To visualize the periodicities of the NN and NNN interactions, we show graph representations of these 


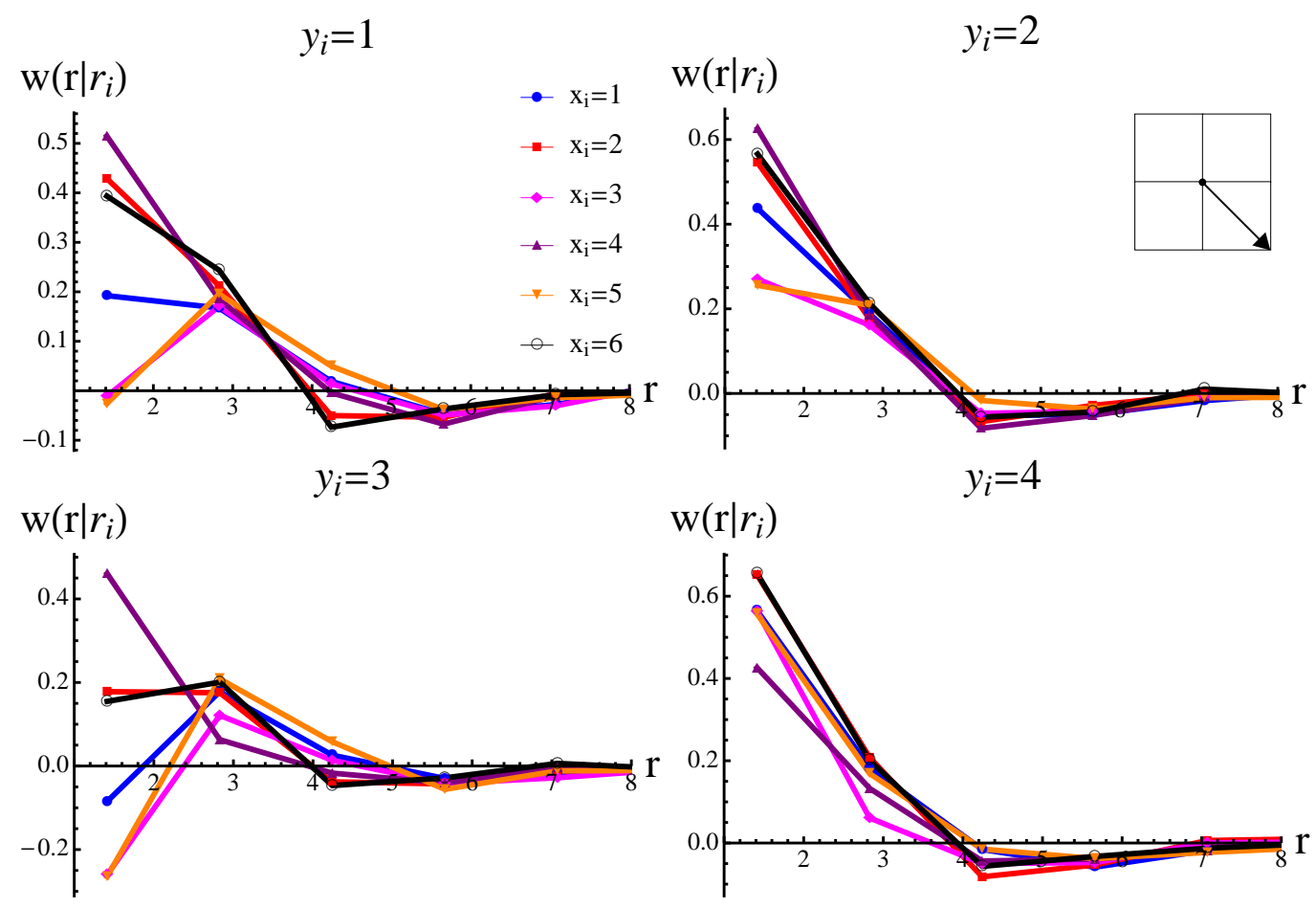

Fig. 11. (Color online) Plots of interactions $w\left(r \mid \boldsymbol{r}_{i}\right)$ against distance $r_{i j}=\sqrt{2} s$ with $s=1,2, \cdots 6$ along the downward slope of the 45-degree angle from fixed $\boldsymbol{r}_{i}$. The same images and parameters as in Fig. 10 are used.

interactions in Fig. 12, employing the fact that the signs of the interactions are different among the sublattices, namely, the positive and negative interactions are colored by blue and red links, respectively. Fig. 12 clearly exhibits the periodicity of the NN and NNN interactions and we can see that a checker board-like structure in the interacting network emerges, which is consistent with the sublattice structure shown in the left and center panels of Fig. 7. Deviations from the checker board structure are also observed, which introduce frustration into the system. However, the number of frustrated plaquettes is not large and we expect the effect of the frustration to be small, meaning the nature of the ground state is simple. If we take into account the NNN interactions, frustration can be enhanced by the antiferromagnetic NNN interactions in plaquettes consisting of four antiferromagnetic NN interactions, but the effect on the ground state is again expected to be weak, since these antiferromagnetic NNN interactions are small in absolute value as compared to other NN and NNN interactions, which is confirmed in the histogram of the NN and NNN interactions, $P\left(w_{i j} \mid r_{i j}=1\right)$ and $P\left(w_{i j} \mid r_{i j}=\sqrt{2}\right)$, given in Fig. 13. The histograms clearly reflect the sublattice structure shown in Fig. 7. The insets show the reduced versions of the left and center panels of Fig. 7. Multiple peaks observed in the NN interactions, the magenta part in the left panel of Fig. 13, imply another additional periodicity in the NN interactions, but we do not pursue this point to avoid complexity, as we declared in sec. 3.1.1. 

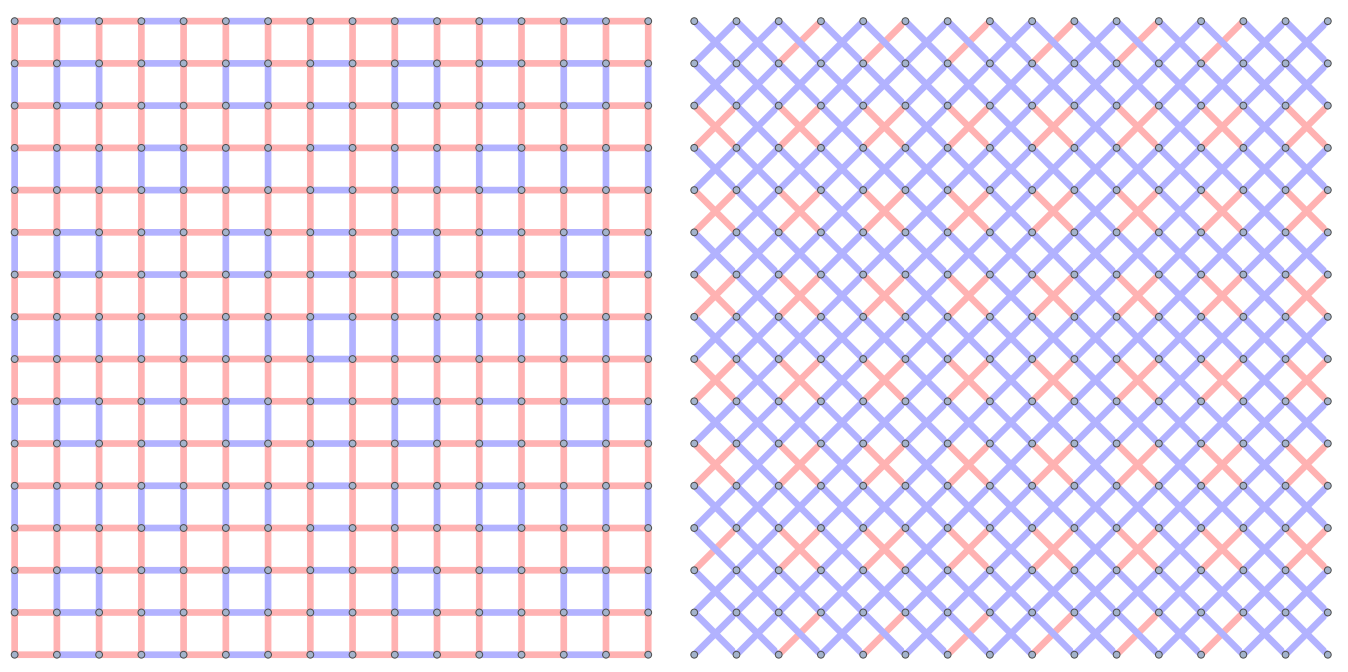

Fig. 12. (Color online) Graph representations of the NN (left) and NNN (right) interactions inferred from the face pictures of patch size $L=16$ plotted in the coordinate space $\boldsymbol{r}=(x, y)$. Each site corresponds to each pixel location and the links correspond to the interactions between the sites. Blue and red links denote positive and negative interactions, respectively. Clear periodicity and a checker board-like structure are observed.
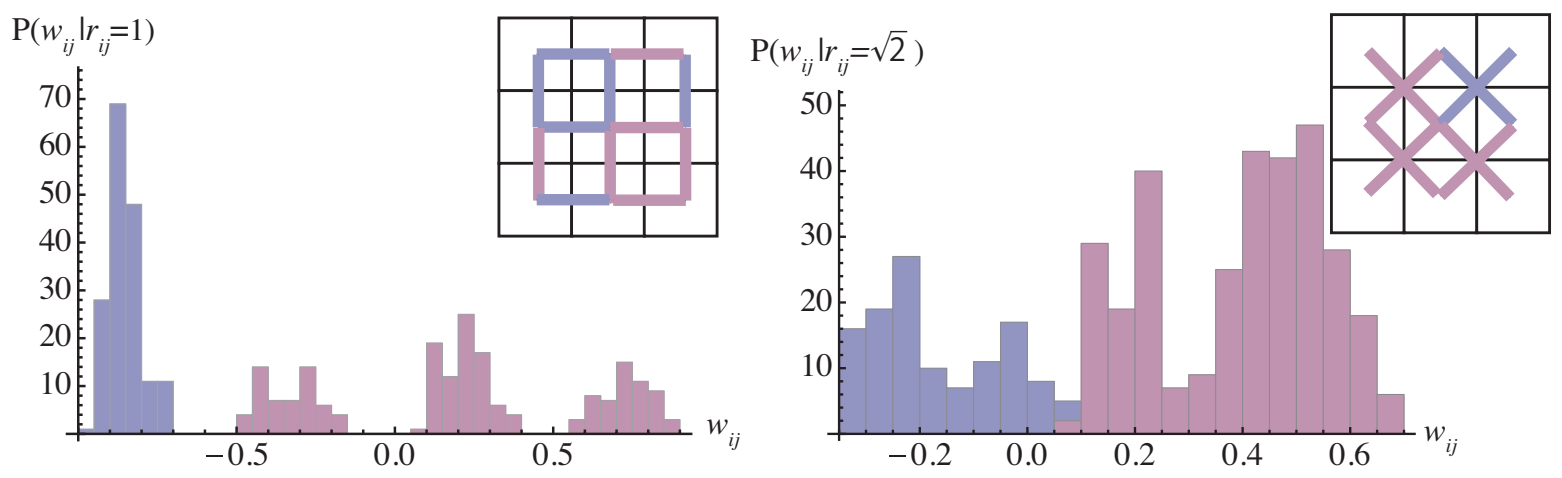

Fig. 13. (Color online) Histograms of the NN (left) and NNN (right) interactions derived by the NMF from face pictures of patch size $L=16$. Each histogram consists of two categories corresponding to the sublattice structure, which is shown in the inset. The colors of the histograms correspond to the two different types of links in the inset.

Although the frustration is weak and possibly does not affect the ground state, some metastable states can emerge because of the frustration and can influence the nature of the system. In, ${ }^{13)}$ the role of these metastable states was discussed in connection with biological visual systems, based on observations that the patterns of the metastable states can be interpreted as filters selecting certain directions of edges discriminating two uniform regions. This may in fact be interesting, but we do not pursue this point since the enumeration of the metastable states is not easy in our case, because the system size is significantly larger than that in; ${ }^{13)}$ however, additional remarks are presented in sec. 4. 
Further, we examine the finite size effects and the difference between the NMF and BA. For this, we plot $\overline{w_{A}}(r)$ and $\overline{w_{B}}(r)$, the definitions of which are the same as in the case of aerial pictures, for different patch sizes $L=8,16$, and 32 in Fig. 14. This figure shows that the size effect is again absent
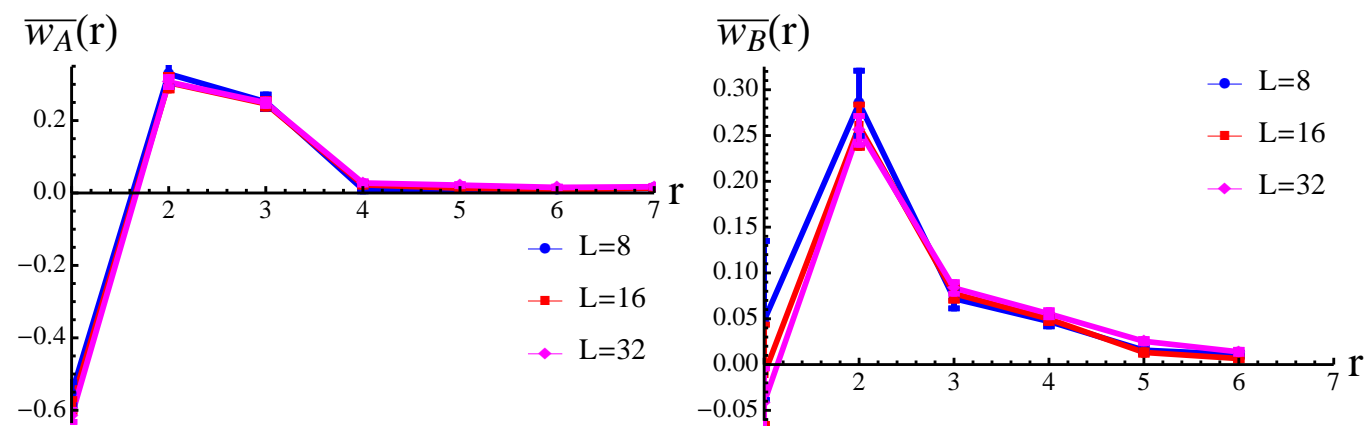

Fig. 14. (Color online) Plots of $\overline{w_{A}}(r)$ (left) and $\overline{w_{B}}(r)$ (right) for different patch sizes $L=8,16$, and 32 . The number of used patches is $B=1,536,384$ and 174,096 for $L=8$ and 32, respectively. The size effect is quite tiny.

and the characteristic length scale is about $\xi \approx 4$. Similar plots to compare the NMF and BA are given in Fig. 15. We again see that almost no difference exists between the NMF and BA. Thus, the findings

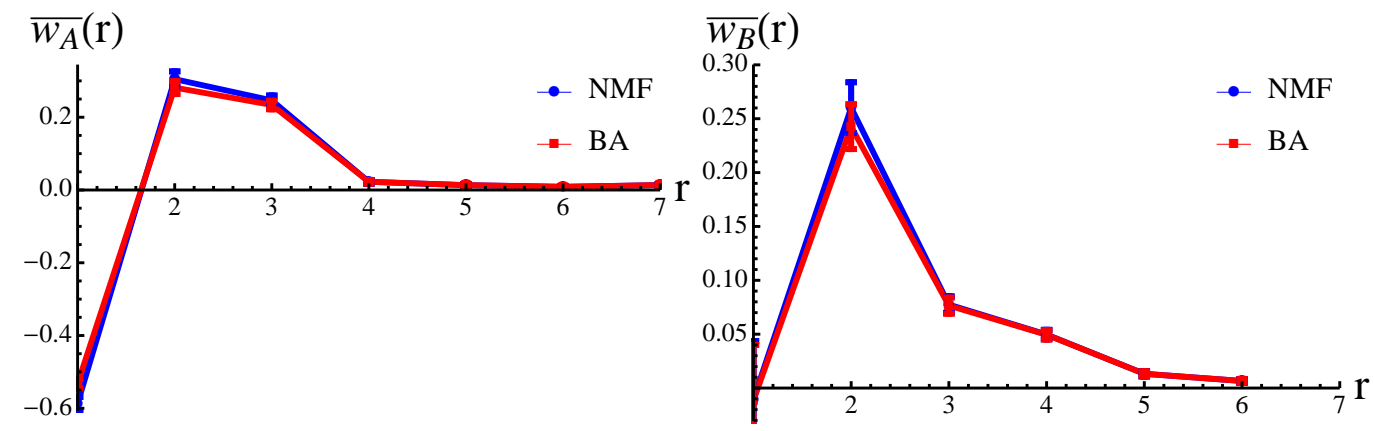

Fig. 15. (Color online) Comparison in $\overline{w_{A}}(r)$ (left) and $\overline{w_{B}}(r)$ (right) between the NMF and BA for patch size $L=16$. The difference is negligible.

by the NMF for patch size $L=16$ are expected to hold.

\subsubsection{Forest Pictures}

Let us move to the case of the forest pictures. The interactions in a row inferred by the NMF are plotted in Fig. 16, as Fig. 5 and Fig. 10. The interaction range, boundary effect, and periodicities are common, as in the previous two cases. As in Figs. 6 and 11, the interactions along the downward slope of the forest pictures are displayed in Fig. 17. The behavior is similar to that in the aerial picture case and the periodicity is not clearly seen in the NNN interactions. The graph representations of the 

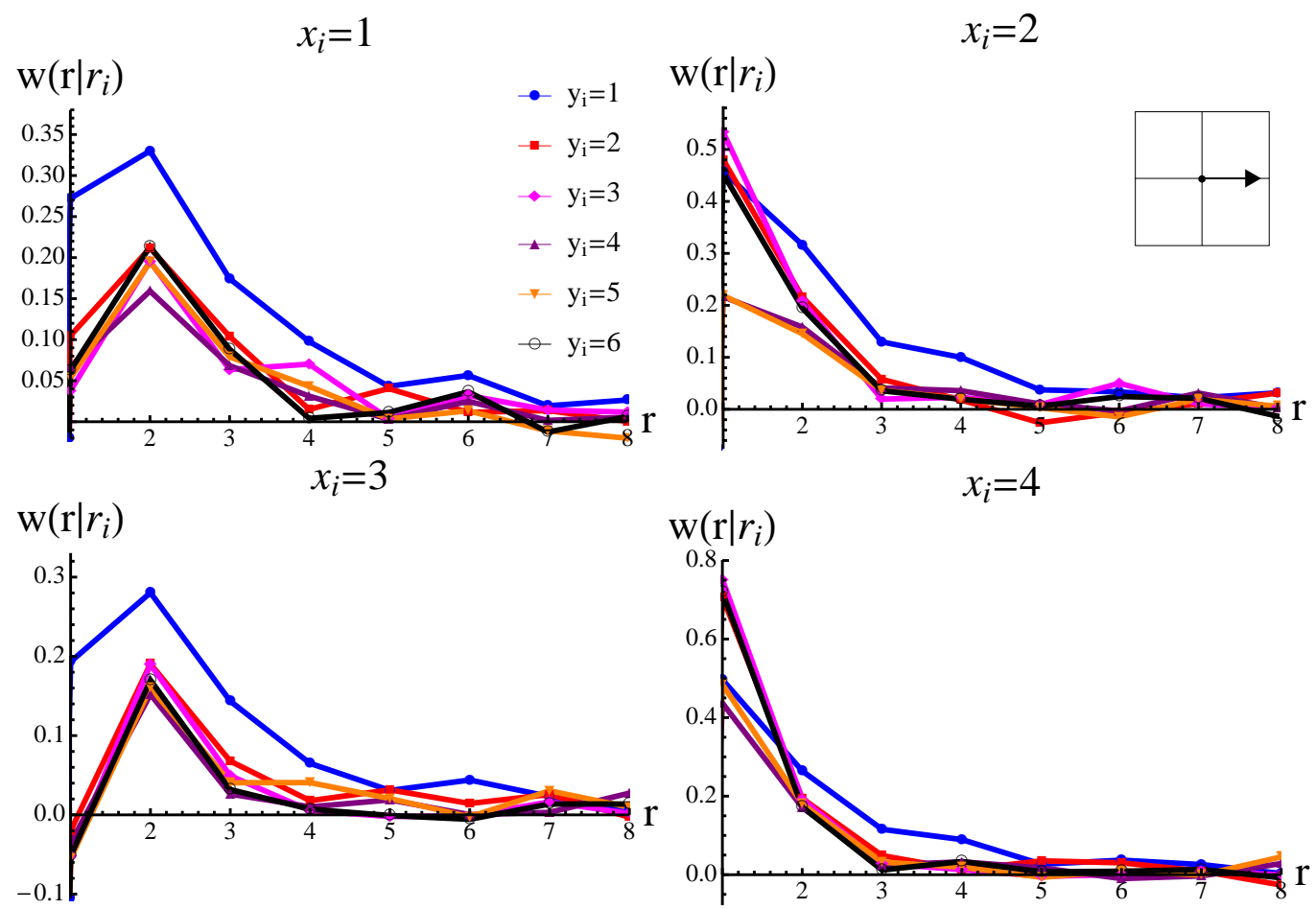

Fig. 16. (Color online) Plots of interactions $w\left(r \mid \boldsymbol{r}_{i}\right)$ against distance $r=\left|x_{j}-x_{i}\right|$ in a common row $y_{i}=y_{j}$ as the origin of the plot $\boldsymbol{r}_{i}=\left(x_{i}, y_{i}\right)$ changes (the top-right inset represents the moving direction of $\left.\boldsymbol{r}_{j}\right)$. The interactions are inferred by the NMF using $B=576,096$ patches of size $L=16$ created from forest pictures.
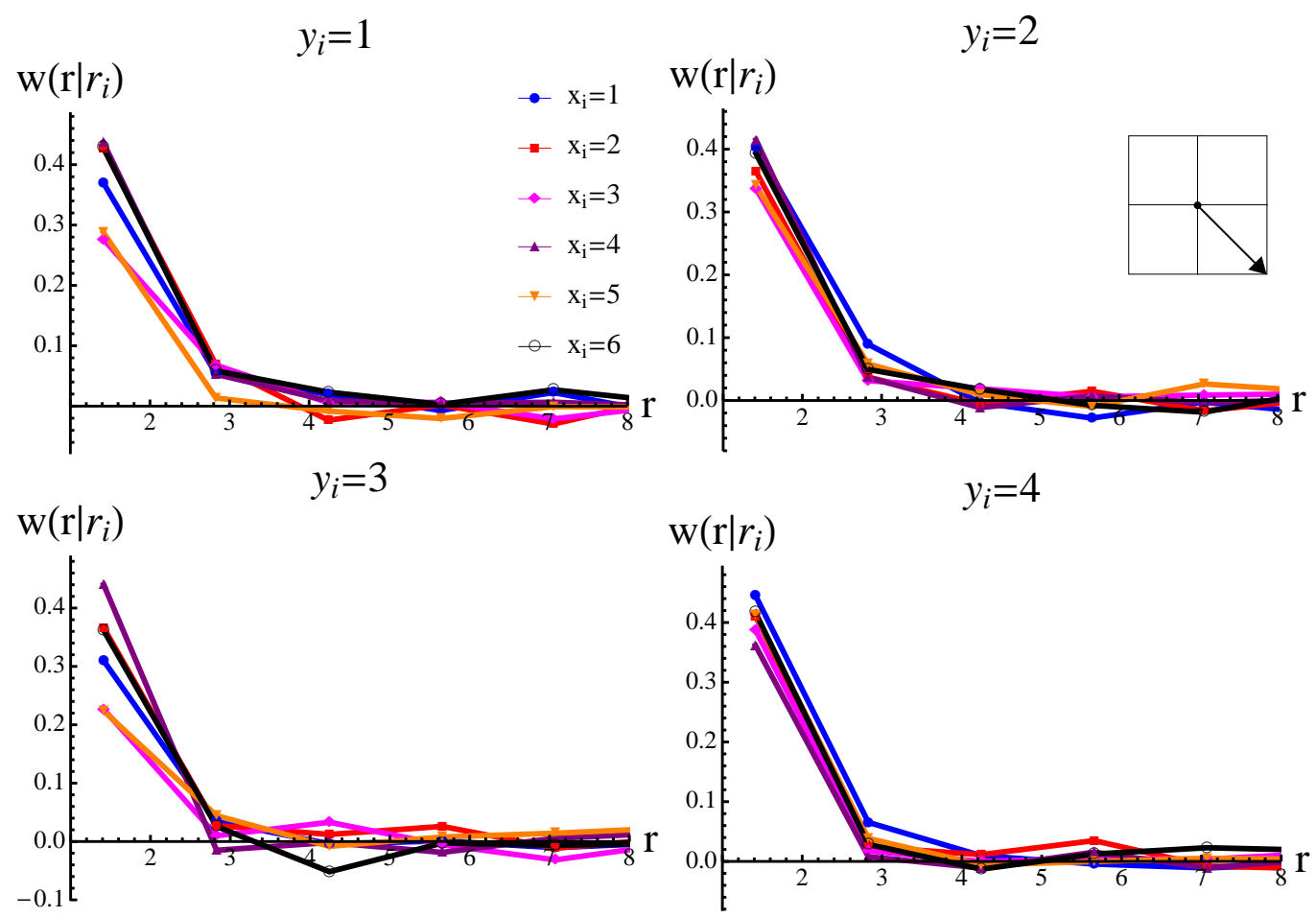

Fig. 17. (Color online) Plots of interactions $w\left(r \mid \boldsymbol{r}_{i}\right)$ against distance $r_{i j}=\sqrt{2} s$ with $s=1,2, \cdots 6$ along the downward slope of the 45-degree angle from fixed $\boldsymbol{r}_{i}$ (the top-right inset represents the moving direction of $\boldsymbol{r}_{j}$ ). The same images and parameters as in Fig. 16 are used. 
NN and NNN interactions are shown in Fig. 18, corresponding to Fig. 12. The checker board-like
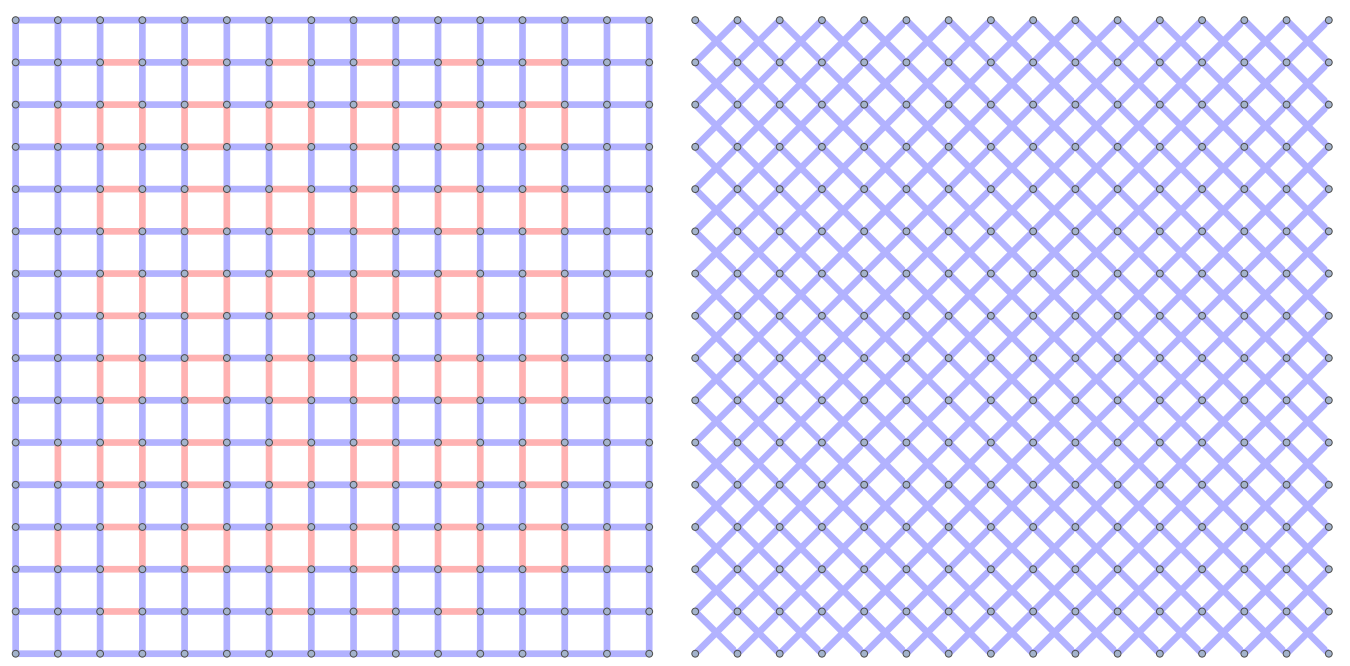

Fig. 18. (Color online) Graph representations of the NN (left) and NNN (right) interactions inferred from the forest pictures of size $L=16$ plotted in the coordinate space $\boldsymbol{r}=(x, y)$. Checker board-like structures are observed in the NN interaction network, except for in the boundaries.

structure is again observed in the $\mathrm{NN}$, but not in the NNN interaction network, indicating the absence of frustration. Quantitative information about the NN and NNN interactions is obtained from the histograms in Fig. 19. The sublattice structure given in Fig. 7 is again observed in the NN interactions,
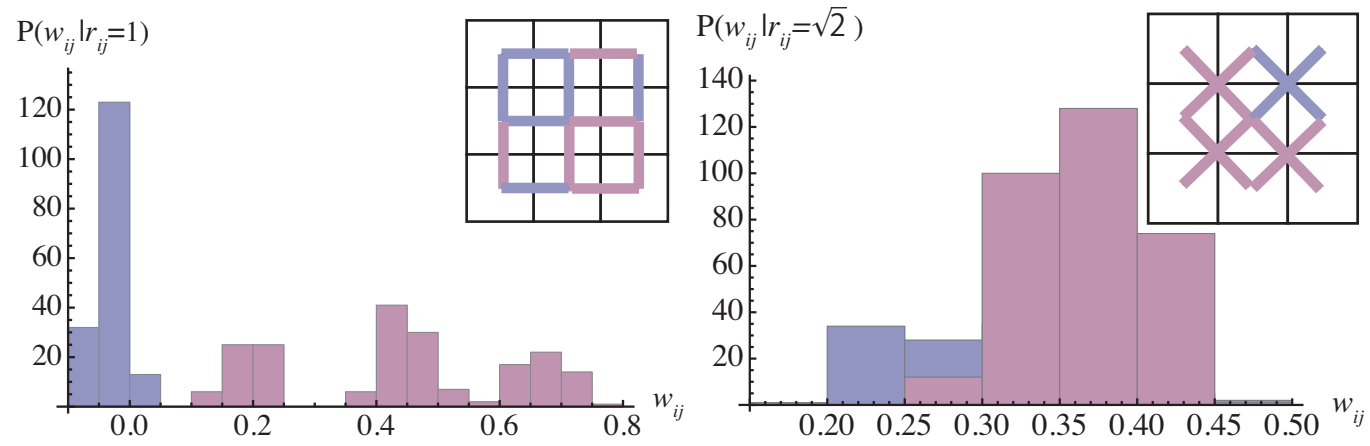

Fig. 19. (Color online) Histograms of the NN (left) and NNN (right) interactions derived by the NMF from forest pictures of patch size $L=16$. The interactions are characterized based on Fig. 7, but no clear difference is observed in the NNN interactions.

including the additional periodicity, in common with the face pictures, signaled by the multiple peaks in the histogram.

The finite-size effect is examined in Fig. 20. In comparison with the previous two cases, we can 

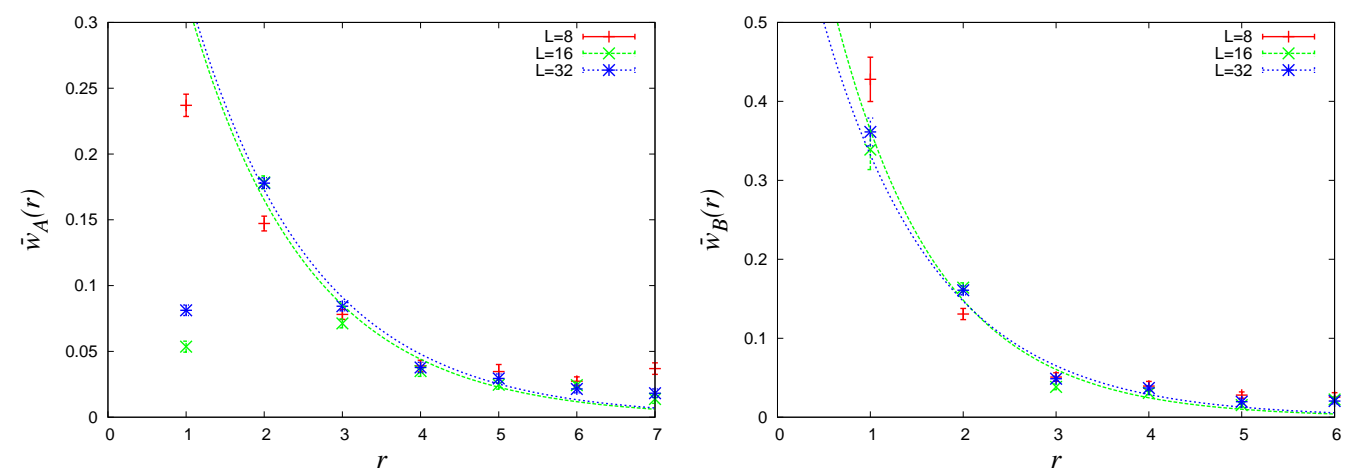

Fig. 20. (Color online) Plots of $\overline{w_{A}}(r)$ (left) and $\overline{w_{B}}(r)$ (right) for different patch sizes $L=8,16$, and 32 . The number of used patches is $B=1,536,384$ and 174, 096 for $L=8$ and 32, respectively. The interactions tend to be longer range than in the cases of aerial and face pictures, which appear to lead to a finite size effect at $L=8$. The curves of the exponential fit to the region $2 \leq r \leq 6$ are given for $L=16$ and 32 .

see that the curves of $\overline{w_{A}}(r)$ and $\overline{w_{B}}(r)$ do not disappear completely around $r \approx 4$, and appear to be longer ranged. A finite-size effect is observed at $L=8$, where the values of interactions at $r=1$ and 7 are larger than for the other two patch sizes, $L=16$ and 32. These facts imply the range of interactions for the forest pictures is longer than in the cases of aerial and face pictures. To examine this point, we attempt the following exponential fit with two parameters $a$ and $b$ :

$$
\bar{w}(r)=a e^{-(r-2) / b} .
$$

The fitting is performed against the region $2 \leq r \leq 6$ for $L=16$ and 32; the resultant curves are given in Fig. 20. The estimated parameters are summarized in Table I. At least visually, the exponential fit is

Table I. Parameters fitted to eq. (16) for forest pictures corresponding to Fig. 20

\begin{tabular}{|l|l|l|l|}
\hline \hline Sublattice & Size $L$ & $a$ & $b$ \\
\hline \hline A & 16 & $0.16 \pm 0.02$ & $1.52 \pm 0.25$ \\
\hline B & 16 & $0.15 \pm 0.03$ & $1.11 \pm 0.28$ \\
\hline A & 32 & $0.17 \pm 0.01$ & $1.56 \pm 0.17$ \\
\hline B & 32 & $0.15 \pm 0.03$ & $1.23 \pm 0.28$ \\
\hline
\end{tabular}

good, and the estimated parameter $b$ takes values around 1.1-1.6. This implies that the interactions in this case are seemingly longer-reached but still rapidly decaying, and it suffices to consider the range $r \leq \xi=2+b \approx 4$, which is in accordance with the cases of aerial and face pictures.

A comparison of the NMF and BA is given in Fig. 21. We observe that the NMF gives larger values of $\bar{w}(r)$, which in particular is clear in the NN interaction of $\overline{w_{B}}$. This tendency was absent for 

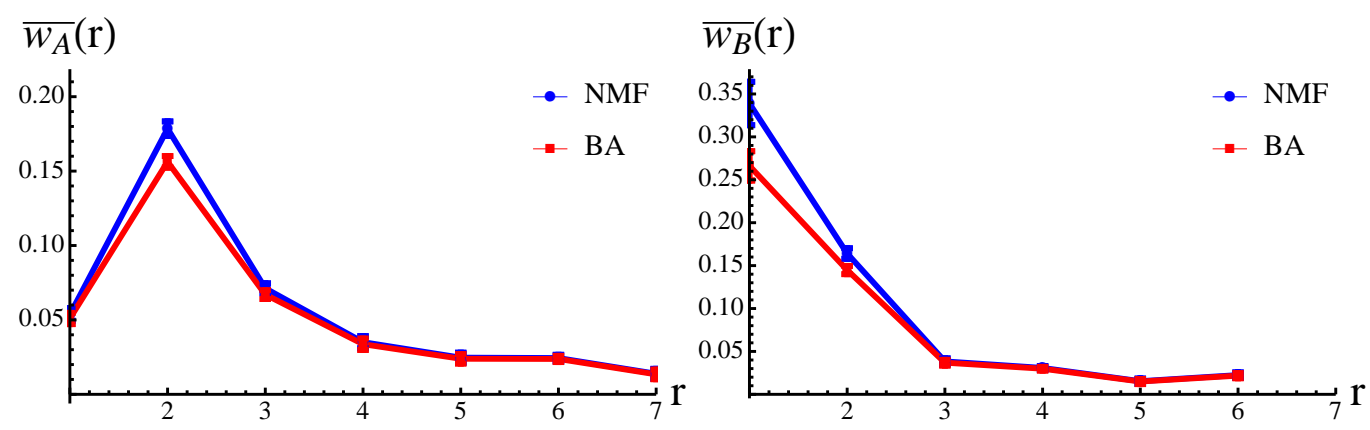

Fig. 21. (Color online) Comparison in $\overline{w_{A}}(r)$ (left) and $\overline{w_{B}}(r)$ (right) of the NMF and BA for patch size $L=16$. Relatively large differences are present at $r=2$ for $\overline{w_{A}}$ and at $r=1$ for $\overline{w_{B}}$.

the previous two sets of pictures and may be related to the fractal nature of the forest pictures, which can be connected to the strong criticality.

\subsection{Robustness of the Results}

For checking the robustness of the results thus far, in this section we present the result by the Monte Carlo (MC) simulation, as well as the result for other dithering methods. For simplicity, we only treat face pictures in this section, but we confirmed that the similar conclusion is obtained for other sets of images.

\subsubsection{Comparison with Monte Carlo Simulation}

The MC simulation is computationally demanding and only the size $L=8$ is treated here. The computation procedure is as follows. We search the maximum of log likelihood, eq. (8), by the Newton method, and the required average over the model in the moment matching condition is computed by the MC method. In the MC method, the well-known Metropolis algorithm is used, and the MC steps for sampling is fixed to be $N_{m c}=10000$. Once the absolute value of the gradient is smaller than a threshold value, here it is chosen to be $10^{-6}$, we regard the algorithm converges and the corresponding $\boldsymbol{w}$ and $\boldsymbol{h}$ are returned as the solution.

The Fig. 22 are the plots of $\overline{w_{A}}(r)$ and $\overline{w_{B}}(r)$ for the NMF, BA and MC methods. This figure clearly shows the functional form of $w$ of the MC result is similar to the ones of the other two methods, though its absolute value of $w$ tends to be smaller. Besides, Fig. 23 displays the graph representation and the histogram of NN interactions. The result is very similar to the one by the mean-field methods given in Figs. 12 and 13. Hence, these finding well supports our main claim, the emergence of sublattice structure and the presence of characteristic length scale $\xi \approx 4$. 

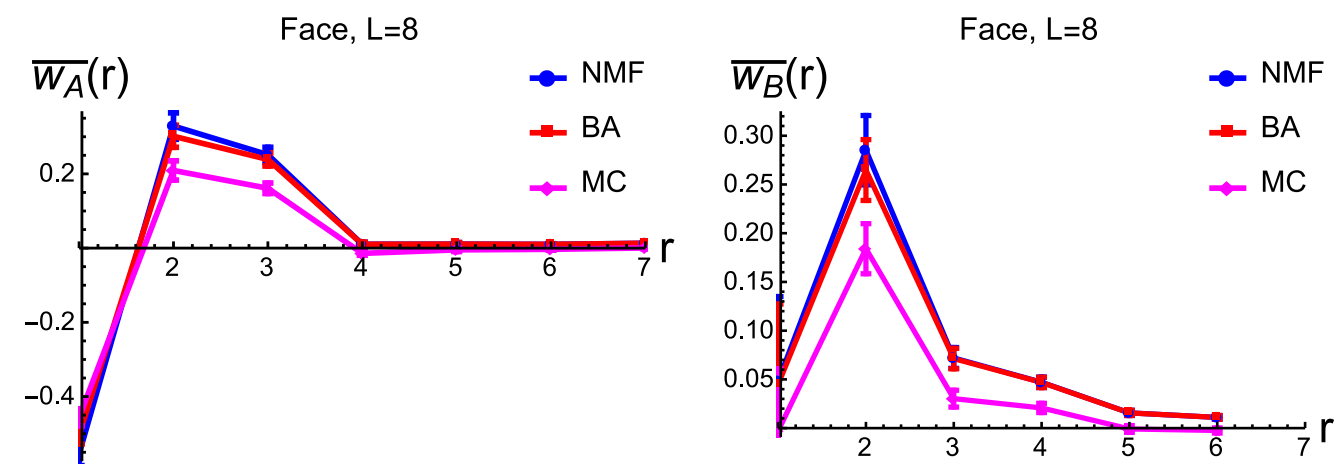

Fig. 22. (Color online) Comparison in $\overline{w_{A}}(r)$ (left) and $\overline{w_{B}}(r)$ (right) of the NMF, BA and MC methods, for patch size $L=8$ of face pictures. The functional shape of the MC result is similar to the other twos but the absolute value of $w$ tends to be smaller.
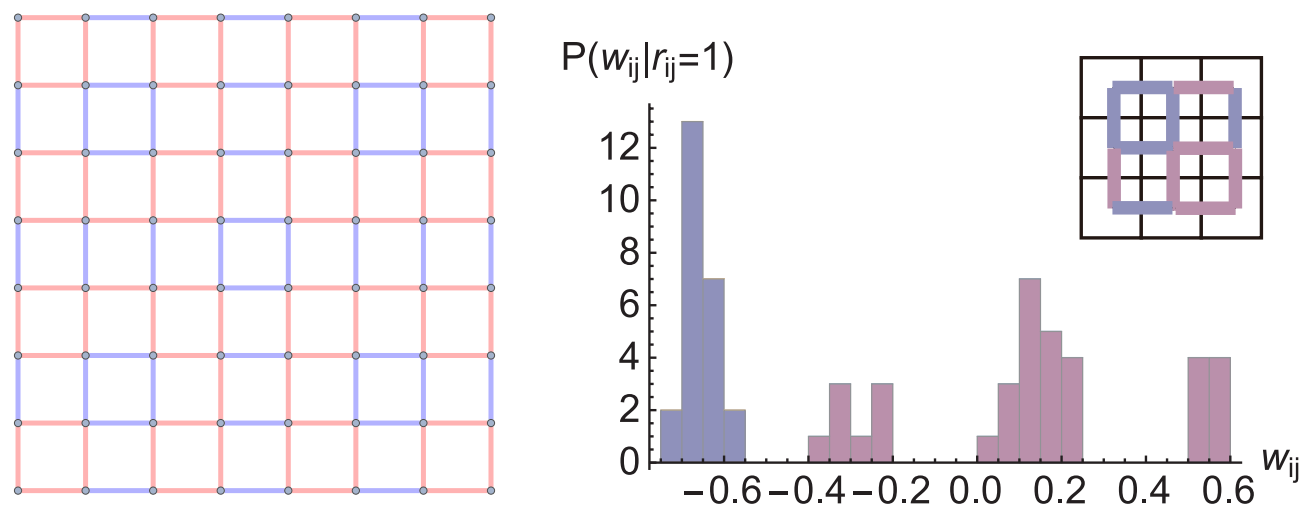

Fig. 23. (Color online) Graph representation (left) and the histogram (right) of the NN interactions derived by the MC simulation from face pictures of patch size $L=8$. The behavior is quite similar to the ones by the NMF in Figs. 12 and 13.

\subsubsection{Other Dithering Methods}

The presented results thus far can be affected by the dithering method employed in preprocessing. Here we examine how the results change if other dithering methods are employed. The inferred method is fixed to be NMF.

There are so many dithering methods such as Floyd, Jarvis, Stucki, Burkes, and Sierra dithering. It is not easy to check all of them, and here we test only the cases with Floyd and no dither.

Fig. 24 shows graph representations of the NN and NNN interactions for the case of Floyd dither. The sublattice structure is clearly matching to the one in Fig. 7, and hence our observation thus far can be applied to Floyd dither. Meanwhile, the result with no dither is given in Fig. 25. Unfortunately, the NNN interaction network does not match to the sublattice structure studied thus far. This difference in the no-dither case from other cases may be understood as follows. Any dither process introduces 

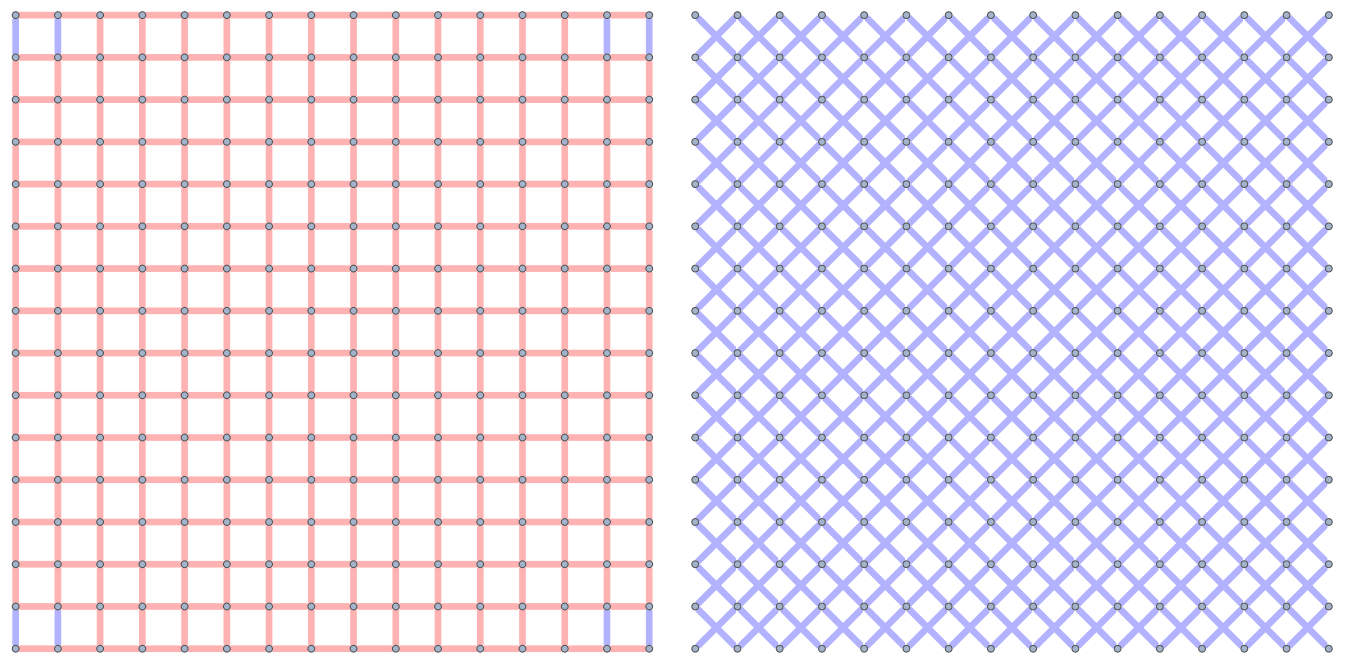

Fig. 24. (Color online) Graph representations of the NN (left) and NNN (right) interactions in Floyd dithering for face pictures of patch size $L=16$. The sublattice structure is matching to the structure in Fig. 7, but is simpler than the one by Riemersma dither shown in Fig. 12.
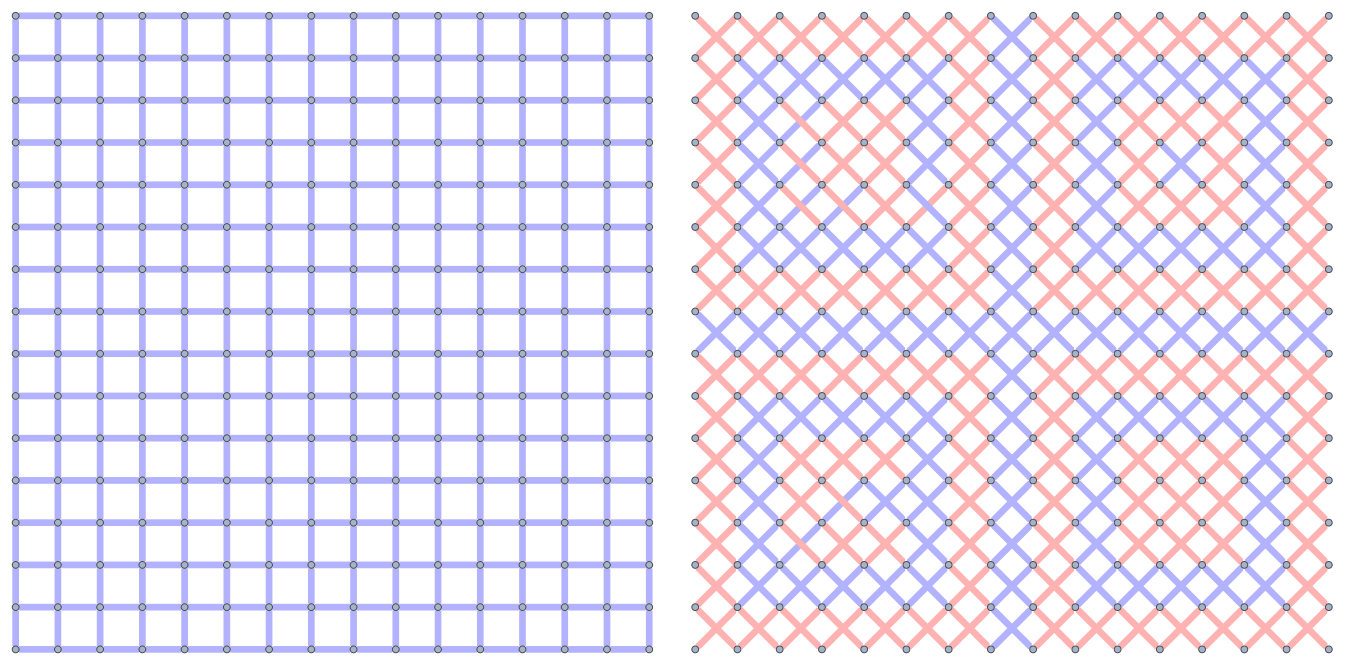

Fig. 25. (Color online) Graph representations of the NN (left) and NNN (right) interactions without any dither for face pictures of patch size $L=16$. The sublattice structure for the NNN interactions is not matching to the structure in Fig. 7.

certain periodicity in the processed pictures to make contrasts among macroscopically discriminable regions. The induced periodicity is expected to have a relatively high frequency or short wavelength, to make the contrast vivid. Our proposed sublattice structure in Fig. 7 has the wavelength 2 (in unit of the number of pixels), which is the nontrivial sublattice structure with the minimum wavelength. Hence based on this reasoning, it is natural that the dither images have this sublattice structure, while images without dither may not have such a contrast sublattice structure. 
Let us move to a quantitative comparison among different dithers. Fig. 26 shows $\overline{w_{A}}(r)$ and $\overline{w_{B}}(r)$ for Riemersma and Floyd dithers. As understood from Fig. 24, the result of Floyd dither has no

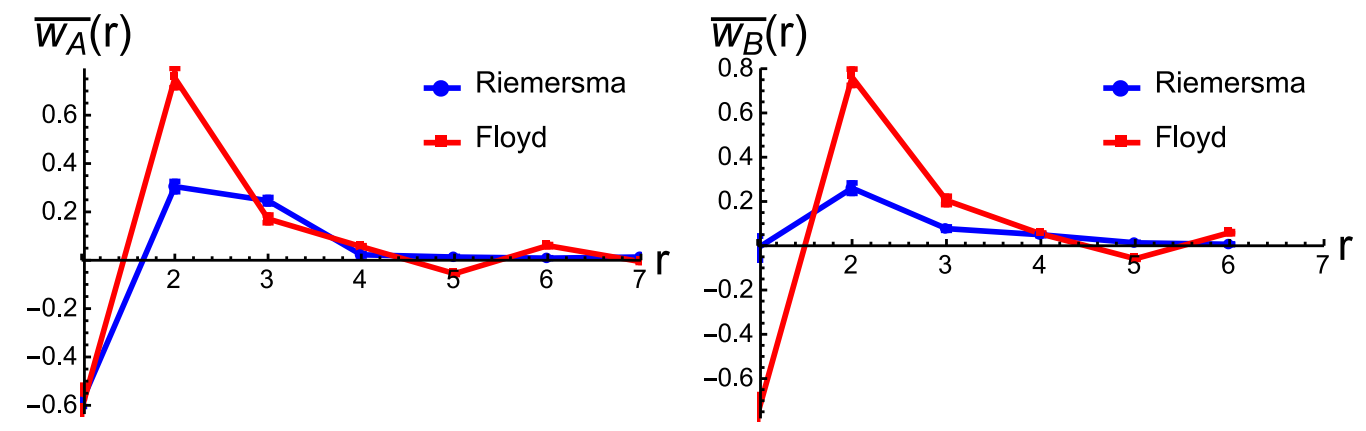

Fig. 26. (Color online) Comparison in $\overline{w_{A}}(r)$ (left) and $\overline{w_{B}}(r)$ (right) of the Riemersma and Floyd dithers, for patch size $L=16$ of face pictures.

difference between $\overline{w_{A}}(r)$ and $\overline{w_{B}}(r)$, since it has a simpler structure. The absolute values of $w$ tend to be larger in Floyd dithering, although in both dithers the interactions rapidly decay as $r$ grows, and almost vanish around $r \approx 4$. The same plot for the no dither case is in Fig. 27. In this case, the value
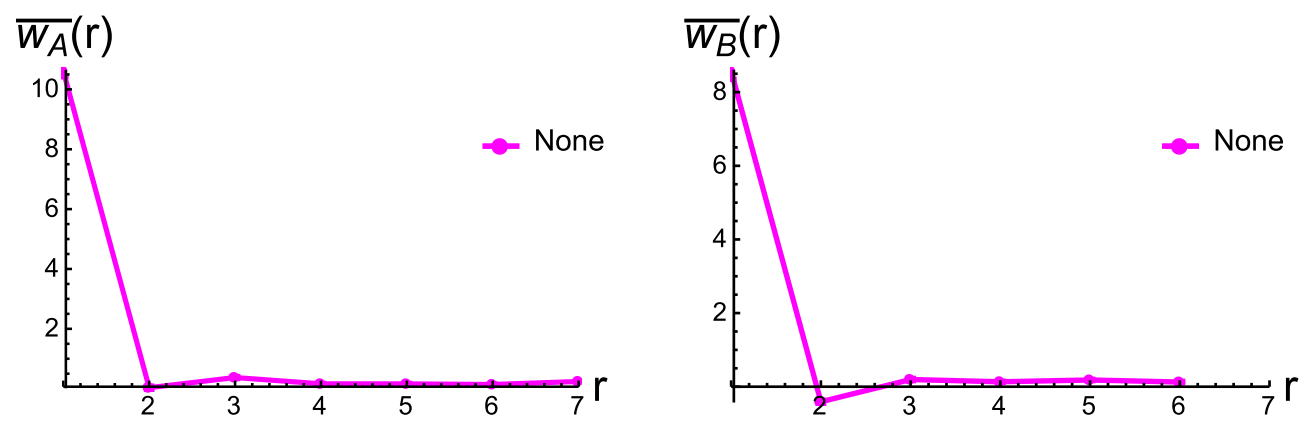

Fig. 27. (Color online) Plots of $\overline{w_{A}}(r)$ (left) and $\overline{w_{B}}(r)$ (right) for patch size $L=16$ of face pictures in the no dither case.

of $w(r)$ at $r=1$ is significantly larger than the dither cases, while it more rapidly decays as $r$ grows (it almost vanishes at $r=2$ ). This might be because in the no dither case any meaningful structure common in any patch of images is absent and only the NN interaction $w(r=1)$ holds the information of the images.

\subsection{Inferred Fields}

In this section, we present the histograms of local magnetizations, $P\left(m_{i}\right)$, and of local fields, $P\left(h_{i}\right)$, inferred by the NMF and the MC simulation, in each set of pictures. We show the results only for $L=16$, since we did not find any meaningful size effect. 
Fig. 28 shows the histograms of local magnetizations of aerial (left), face (center), and forest pictures (right). All the histograms have a simple peak structure in the positive $m_{i}>0$ region. This
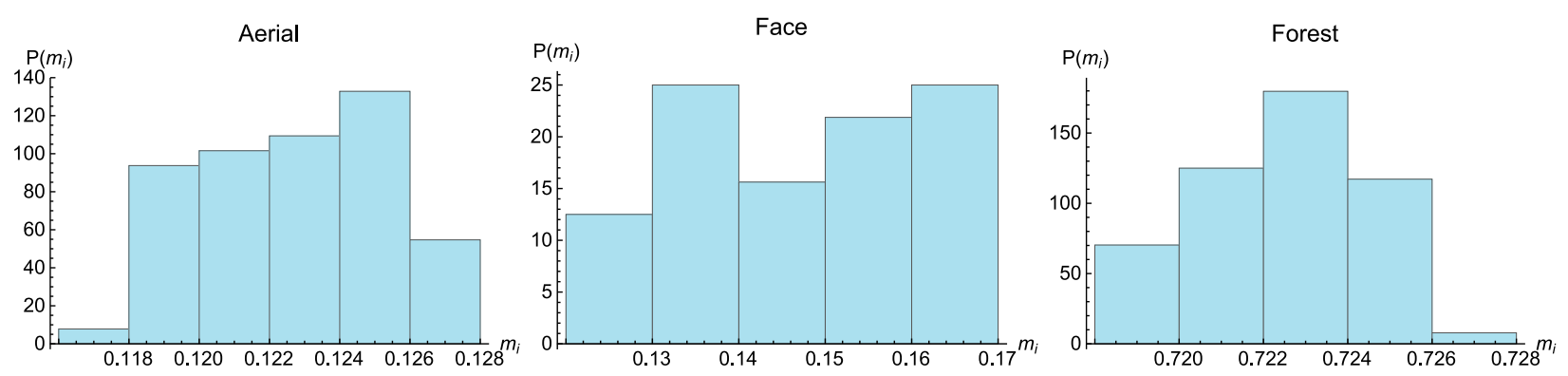

Fig. 28. (Color online) Normalized histograms of the local magnetizations of aerial (left), face (center), and forest pictures (right) for patch size $L=8$. They are normalized as probability distribution, namely, the volume of the histogram is unity. In all cases, the magnetizations are positive, meaning that black pixels appear more frequently.

implies black pixels appear more frequently in all the sets of pictures.

In Fig. 29, we show the histograms of the local fields inferred by the NMF for aerial (left), face (center), and forest pictures (right). The same histograms for the MC simulation are also given in Fig. 30. These figures exhibit the inferred fields tend to be negative for some cases, while the local
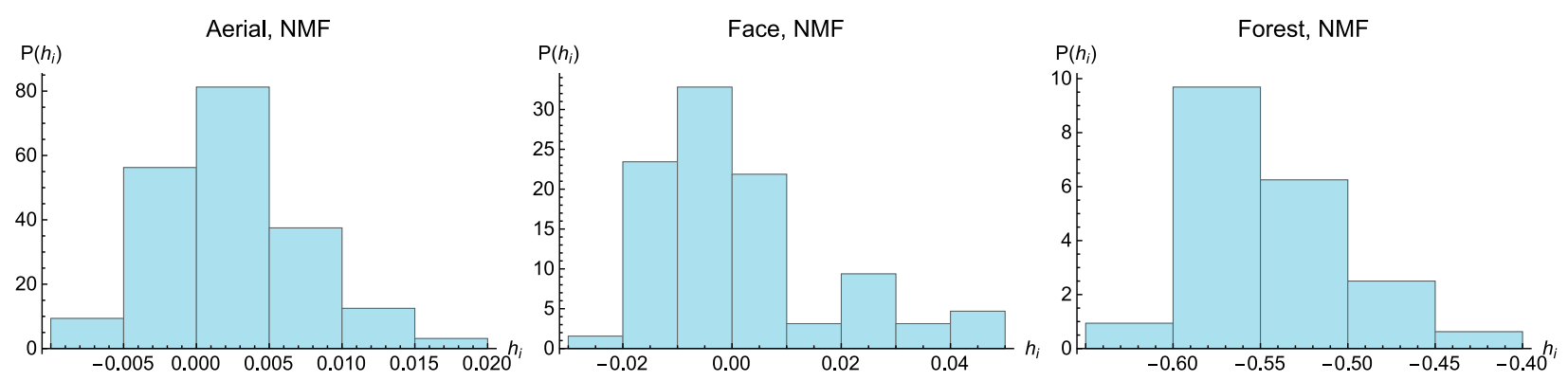

Fig. 29. (Color online) Normalized histograms of local fields of aerial (left), face (center), and forest pictures (right), inferred by the NMF. Although the magnetizations are positive, the local fields inferred by the NMF tend to be negative.

magnetizations are positive. We expect that this seemingly unnatural behavior is a consequence of the higher order statistics of natural images than the second. This can be understood by seeing an example. Suppose pictures are generated from the following simple Boltzmann distribution with $r$ body interactions:

$$
p_{r}=\frac{1}{Z_{r}} e^{-H_{r}(\boldsymbol{S})}, \mathcal{H}_{r}(\boldsymbol{S})=-N K\left(\frac{1}{N} \sum_{i} S_{i}\right)^{r} .
$$



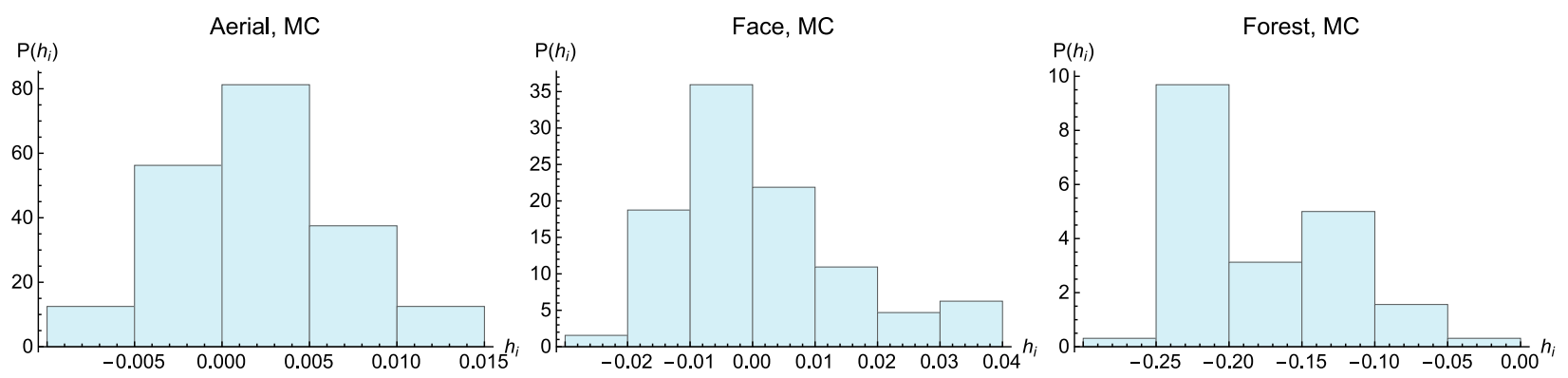

Fig. 30. (Color online) Normalized histograms of local fields of aerial(left), face (center), and forest pictures (right), inferred by the MC simulation. The behavior is similar to the NMF one.

In this case, it is possible to show the mean-field result is exact in the limit $N \rightarrow \infty$, and also possible to derive an analytical formula of the effective pairwise interaction and the local field of eqs. $(9,10)$ in the limit $B \rightarrow \infty$. The computation is straightforward and we only refer to the result:

$$
w_{i j}=\frac{K}{N} r(r-1) m^{r-2}, h_{i}=-K r(r-2) m^{r-1},
$$

where $m$ is the spontaneous magnetization of eq. (17) and is the solution of the following selfconsistent equation

$$
m=\tanh \left(K r m^{r-1}\right) .
$$

Eq. (18) tells us that the sign of the local field changes at $r=2$ and negative for $r>2$ with positive $m$. Hence, it is possible to regard the negativity of inferred fields in Figs. 29 and 30 as the indirect evidence of the importance of high order statistics in natural images.

\section{Discussion}

\subsection{Criticality}

In this section, we address the possible criticality of the Boltzmann machine after learning. The set up of the investigation is as follows. We use the interactions and fields derived by the BA, $\boldsymbol{w}=\boldsymbol{w}_{\mathrm{BA}}$ and $\boldsymbol{h}=\boldsymbol{h}_{\mathrm{BA}}$, for each set of pictures. We define a new Ising model with temperature, the probability distribution of which is

$$
p\left(\boldsymbol{S} \mid \boldsymbol{w}_{\mathrm{BA}}, \boldsymbol{h}_{\mathrm{BA}}, T\right)=\frac{1}{Z\left(\boldsymbol{w}_{\mathrm{BA}}, \boldsymbol{h}_{\mathrm{BA}}, T\right)} e^{-\frac{\mathcal{H}\left(S \mid w_{\mathrm{BA}}, \boldsymbol{h}_{\mathrm{BA}}\right)}{T}},
$$

and hence, the original Boltzmann machine corresponds to $T=1$. We employ a standard MonteCarlo technique to simulate this Ising model, which enables us to calculate physical quantities while changing the temperature. If the original Boltzmann machine is critical, characteristic features in certain physical quantities appear around $T=1$. 
To make the point clearer, we calculate the specific heat ( $N=L^{2}$ is the total number of spins)

$$
C=\frac{1}{N T^{2}}\left(\left\langle\mathcal{H}^{2}\left(\boldsymbol{S} \mid \boldsymbol{w}_{\mathrm{BA}}, \boldsymbol{h}_{\mathrm{BA}}\right)\right\rangle_{\boldsymbol{w}_{\mathrm{BA}}, \boldsymbol{h}_{\mathrm{BA}}, T}-\left\langle\mathcal{H}\left(\boldsymbol{S} \mid \boldsymbol{w}_{\mathrm{BA}}, \boldsymbol{h}_{\mathrm{BA}}\right)\right\rangle_{\boldsymbol{w}_{\mathrm{BA}}, \boldsymbol{h}_{\mathrm{BA}}, T}\right)
$$

and identify the peak location of the specific heat as the "critical" point. Here, the brackets $\langle\cdots\rangle_{w_{\mathrm{BA}}, h_{\mathrm{BA}}, T}$ denote the average over the Ising model. This is a natural choice, since the specific heat is connected to the variation ratio of the entropy as the temperature changes and is known in fact to show a characteristic divergence at the critical point in many systems. The data of the specific heat of the Ising models are shown in Fig. 31. For comparison, on the upper row we display the data with
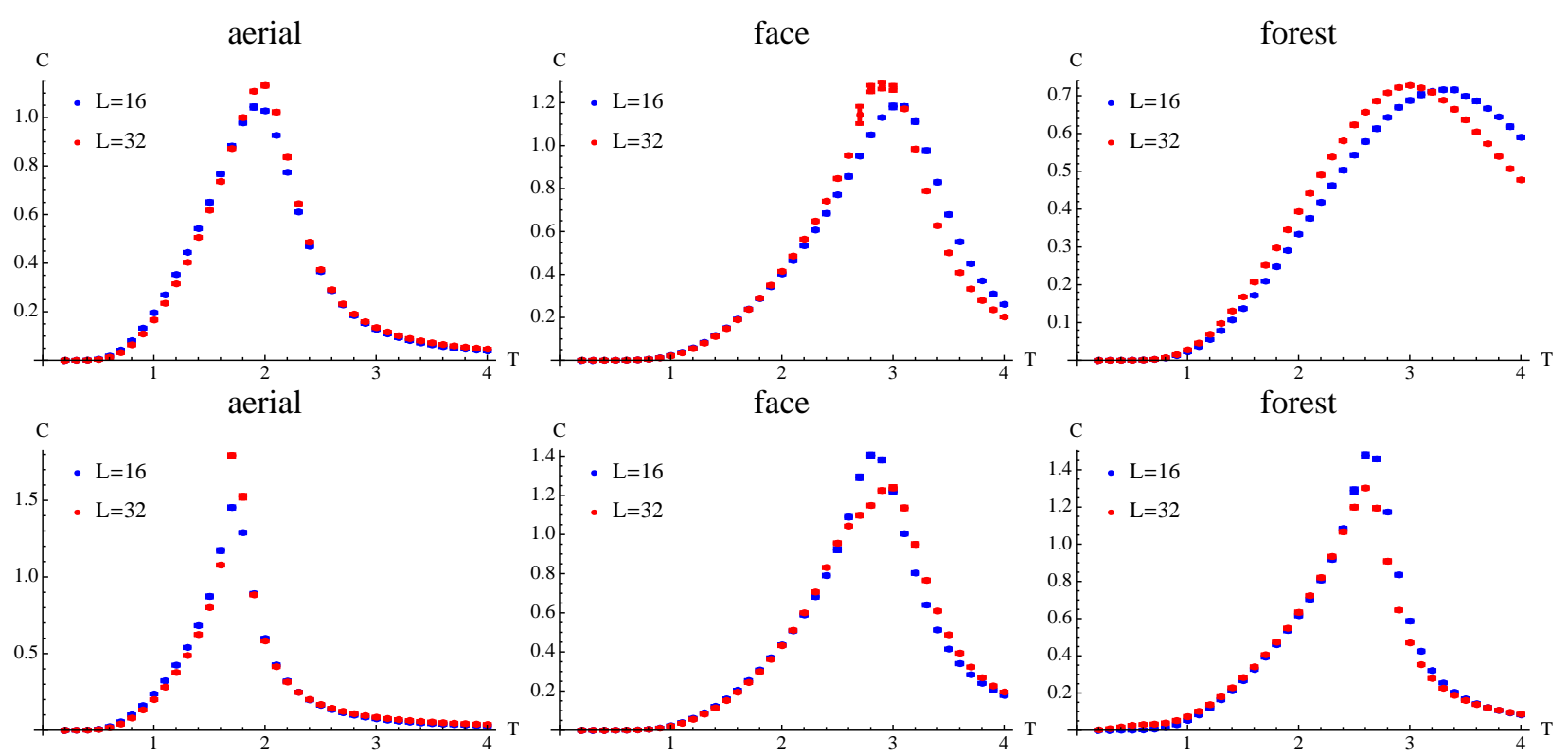

Fig. 31. (Color online) Specific heat of the Ising models defined from the Boltzmann machines derived by the BA analysis of natural images, plotted against the temperature $T$. The data on the upper row are for $\boldsymbol{h}=\boldsymbol{h}_{\mathrm{BA}}$, but on the lower row correspond to $\boldsymbol{h}=\mathbf{0}$. The original Boltzmann machines correspond to $T=1$. Peak locations of the specific heat are far above $T=1$, irrespective of the presence of the on-site fields.

the fields set to be the values inferred by the BA, $\boldsymbol{h}=\boldsymbol{h}_{\mathrm{BA}}$, while on the lower row the data without the fields, $\boldsymbol{h}=\mathbf{0}$, are shown. As seen clearly, the peaks of specific heat locate far above $T=1$ in all the figures, implying that the Boltzmann machines after learning are rather in the lower temperature regions than at the critical point. The data without the fields show sharp peaks and thus these Ising models do in fact enjoy phase transitions. In addition, the Ising model for face pictures shows another complicated structure: we see not only a peak at $T \approx 3.0$ but also a shoulder-like structure at $T \approx 2.6$. The shoulder may be an indication of another transition, which may be similar to the multiple transitions in the Ising model on the Union Jack lattice. ${ }^{27)}$ This seems to be reasonable, since the inferred interactions of the face pictures are similar to those of the Union Jack lattice (see Fig. 12). 
Why does the learned Boltzmann machine not exhibit criticality? There are two possibilities: one is that our binarized images are not at criticality; the other is that the Boltzmann machine cannot extract the criticality of natural images. To examine the first possibility, we plot the Fourier amplitude against the Fourier spatial frequency in Fig. 32 of the aerial pictures as a representative. In the figure,

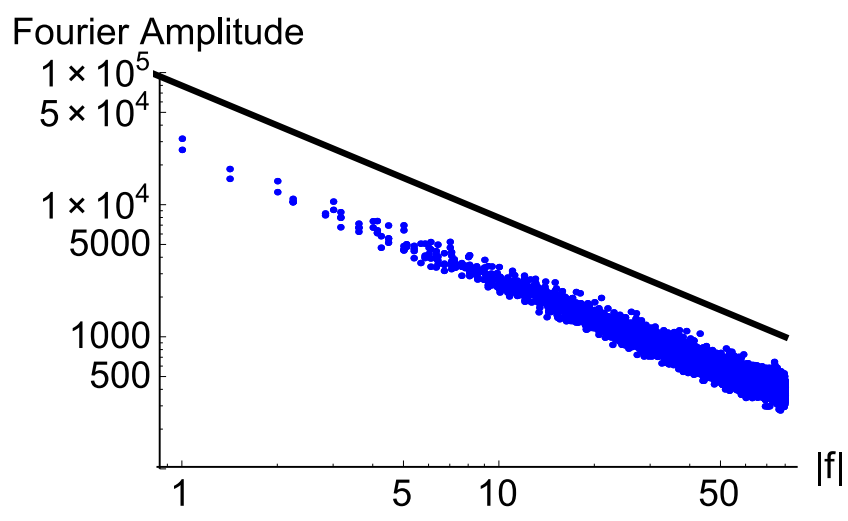

Fig. 32. (Color online) Fourier amplitude of aerial pictures plotted against the absolute value of Fourier spatial frequency. The data show a clear power law comparable with $c /|f|$ (black straight line).

we see a clear power law, even after the pictures are preprocessed by quantization and dithering, indicating that the criticality still holds in our binarized pictures. Similar behavior is seen in the face and forest pictures. Thus, the first possibility, that our binarized pictures are not at criticality, is not the case, and the second, that the Boltzmann machine cannot extract the criticality of natural images, should occur. One possible origin of this defect of the Boltzmann machine is that it does not take into account higher order statistics than the second. It is known that high order statistics exist in natural images and significantly influence the properties of images. ${ }^{5,7)}$ It is thus likely that the source of the criticality of natural images is these high order statistics, which explains why our learned Boltzmann machine does not show criticality, and reinforces the importance of high order statistics.

\subsection{Relation to Simple-Cell Receptive Fields}

It is known that simple-cell receptive fields operate as certain oriented bandpass filters. ${ }^{28)}$ Olshausen and Field naturally derived these filters by processing natural images based on sparse coding. ${ }^{4)}$ In this section, we connect our findings with these known results.

First, we confirm that the oriented bandpass filters are also derived from our binarized pictures after quantization and dithering. We assume that picture $S$ is represented by a linear superposition of 
basis functions $\left\{\boldsymbol{\phi}_{k}\right\}_{k}$ as

$$
S \approx \sum_{k} a_{k} \boldsymbol{\phi}_{k}
$$

and the coefficients $\left\{a_{k}\right\}_{k}$ are sparse (a few non-zero components are needed to express an image) if we choose an appropriate set of basis functions $\left\{\boldsymbol{\phi}_{k}\right\}_{k}$. Olshausen and Field constructed such an appropriate set by solving the following optimization problem

$$
\min _{\boldsymbol{\phi}} \min _{\left\{a_{k}\right\}_{k}}\left(\left\|\boldsymbol{S}-\sum_{k} a_{k} \boldsymbol{\phi}_{k}\right\|_{2}^{2}+R\left(\left\{a_{k}\right\}_{k}\right)\right),
$$

where $R\left(\left\{a_{k}\right\}_{k}\right)$ is an appropriate regularization term to induce the sparsity of the coefficients $\left\{a_{k}\right\}_{k}$. Some typical choices are $R\left(\left\{a_{k}\right\}_{k}\right)=\lambda \sum_{k}\left|a_{k}\right|$ or $R\left(\left\{a_{k}\right\}_{k}\right)=\sum_{k} \log \left(1+a_{k}^{2}\right)$. To follow their way, we use their numerical package called sparsenet. ${ }^{29)}$ According to their method, we whiten our binarized images $\left\{\boldsymbol{S}^{(\mu)}\right\}_{\mu}$ and cut them into patches of an appropriate size (here we choose $8 \times 8$ patches). After these preprocessing, we construct the basis function by using their algorithm.

The result of the construction of the basis function is depicted in Fig. 33. Here 64 different basis
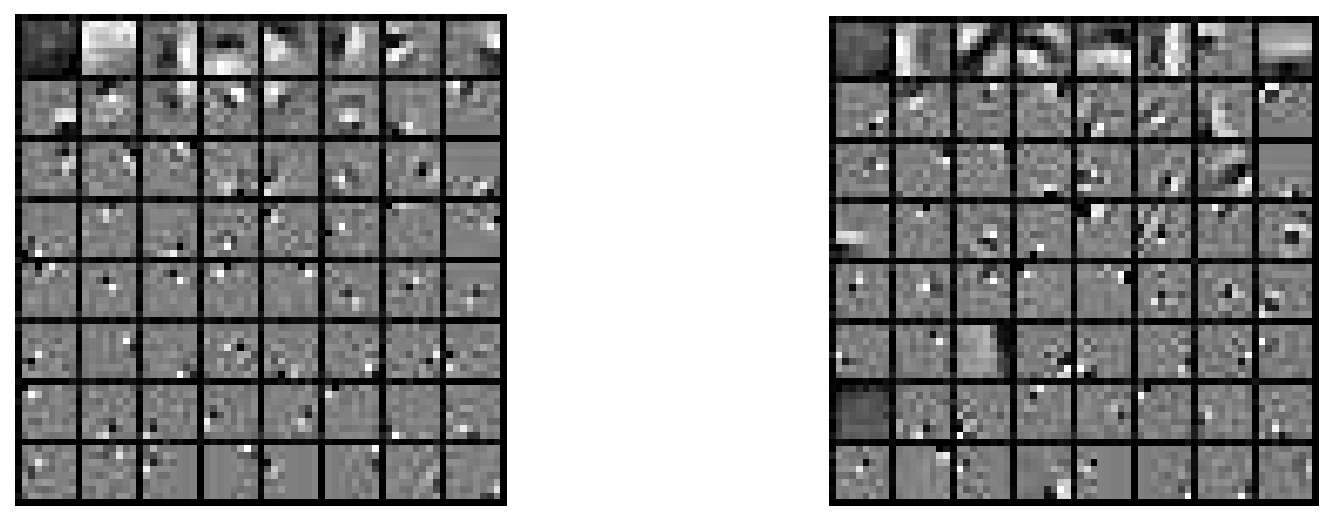

Fig. 33. Results of sparse-coding construction of 64 basis functions estimated from aerial (left) and face pictures (right), both of which are cut into $8 \times 8$-size patches after binarization employing quantization and dithering. Both panels consist of 64 square images each of which represents a basis function. Each basis function has $8 \times 8$ pixels. These two panels are quite similar. Several basis functions exhibit orientations seen as a pair of neighboring white and black pixels.

functions are shown. Each basis function is a square image of $8 \times 8$ pixels in a panel. The results of the two sets of pictures, aerial (left panel) and face pictures (right panel), are very similar. They are also similar to those derived in, ${ }^{4)}$ although our patterns are more localized, which is presumably due to binarization. These results imply that the basis functions of natural images derived by sparse coding are fairly universal. ${ }^{5)}$ Hence, we may assume the presence of an universal set of basis functions that 
can describe any natural image.

The probability distribution of images $P(\boldsymbol{S})$, which is the object of the analysis and approximated by the Boltzmann machine in this study, can be connected to the sparse representation above by the Bayes rule

$$
P(\boldsymbol{S})=\int \prod_{k} d a_{k} P\left(\boldsymbol{S} \mid\left\{a_{k}\right\}_{k},\left\{\boldsymbol{\phi}_{k}\right\}_{k}\right) P\left(\left\{a_{k}\right\}_{k}\right),
$$

where $P\left(\boldsymbol{S} \mid\left\{a_{k}\right\}_{k},\left\{\boldsymbol{\phi}_{k}\right\}_{k}\right)=\delta\left(\boldsymbol{S}-\sum_{k} a_{k} \boldsymbol{\phi}_{k}\right)$ and $P\left(\left\{a_{k}\right\}_{k}\right)$ is the prior distribution of the coefficients. The results of the Boltzmann machine learning indicate that $P(S)$ is different among the different sets of images, implying that $P\left(\left\{a_{k}\right\}_{k}\right)$ is different among these sets of pictures, since the basis functions $\left\{\boldsymbol{\phi}_{k}\right\}_{k}$ are universal. These considerations show that our observation based on the Boltzmann machine captures some characteristics of the prior distribution $P\left(\left\{a_{k}\right\}_{k}\right)$.

The prior distribution $P\left(\left\{a_{k}\right\}_{k}\right)$ should reflect the sparseness of $\left\{a_{k}\right\}_{k}$ and be far from Gaussian. In fact, the high order statistics of natural images originate in the non-Gaussianity of $P\left(\left\{a_{k}\right\}_{k}\right)$. If $P\left(\left\{a_{k}\right\}_{k}\right)$ is a multivariate Gaussian, the corresponding $P(\boldsymbol{S})$ becomes quadratic with respect to $\boldsymbol{S}$, meaning that the Boltzmann machine is sufficient to learn all the characteristics of images; however, this would not be the case as discussed thus far. The appropriate functional form of $P\left(\left\{a_{k}\right\}_{k}\right)$ contains certain hyper parameters reflecting the sparseness of $\left\{a_{k}\right\}_{k}$. Our results based on the Boltzmann machine suggest that such hyper parameters can depend on the choice of images, thus we should change the hyper parameters when processing image data. That is the whole message in the present paper.

Unfortunately, it is not easy to determine the functional form and the hyper parameters of $P\left(\left\{a_{k}\right\}_{k}\right)$ from our results. This would constitute an interesting future work.

\subsection{Summary and Model Proposition}

We summarize our observations thus far:

- The range of interactions is about $\xi \approx 4$, and in the region $r \geq 2$ the interaction is positive and rapidly decays as $r$ grows.

- The sublattice structure shown in Fig. 7 is widely present.

- Boundary effects exist and tend to give larger values of interactions than bulk ones.

- Frustration is absent, or quite weak even when it exists.

- Local fields can be negative even if the magnetizations are biased to be positive. This may be an indirect signal of the importance of high order statistics.

- The criticality of natural images is not captured by the Boltzmann machine, may be because of the lack of higher order statistics than the second. 
According to these findings, we propose a model of the prior distribution of natural images with six parameters:

$$
w_{i j}=w\left(\boldsymbol{r}_{i}, \boldsymbol{r}_{j}\right)=\left\{\begin{array}{cc}
w_{N N}^{1}, & (\text { The NN interaction of blue link) } \\
w_{N N}^{2}, & \text { (The NN interaction of magenta link) } \\
w_{N N N}^{1}, & \text { (The NNN interaction of blue link) } \\
w_{N N N}^{2}, & \text { (The NNN interaction of magenta link) } \\
a e^{-\left|r_{i j}-2\right| / b}, & \text { (otherwise) }
\end{array} .\right.
$$

These six parameters can be adaptively determined according to specific problems, but we admit this is not always easy. For convenience in such situations, we display example values of these parameters estimated through our Boltzmann machine learning for patch size $L=16$ in Table II. In particular,

\begin{tabular}{|c|c|c|c|}
\hline & Aerial & Face & Forest \\
\hline \hline$w_{N N}^{1}$ & 0.07 & -0.85 & -0.03 \\
\hline$w_{N N}^{2}$ & 0.32 & 0.2 & 0.43 \\
\hline$w_{N N N}^{1}$ & 0.24 & -0.14 & 0.3 \\
\hline$w_{N N N}^{2}$ & 0.22 & 0.4 & 0.37 \\
\hline $\mathrm{a}$ & 0.1 & 0.3 & 0.16 \\
\hline $\mathrm{b}$ & 0.7 & 1.1 & 1.3 \\
\hline
\end{tabular}

Table II. Model parameters estimated by our Boltzmann machine learning of natural images of size $L=16$ by the NMF

the NN and NNN interactions, $w_{N N}$ and $w_{N N N}$, are the average values of $w_{i j}$ over the corresponding histograms shown in Figs. 7, 13, and 19. The parameters $a$ and $b$ are estimated through fitting based on eq. (16). Two different values of $a$, as well as of $b$, corresponding to two sublattices are averaged to give the values in Table II. As observed, $w_{N N N}^{1}$ and $w_{N N N}^{2}$ are similar for aerial and forest pictures, in which it is difficult to see clear periodicity in the NNN interactions. This prior with estimation of the parameters is the main result of the present study.

\section{Conclusion}

In this paper, we investigated the prior distributions of natural images by employing the Boltzmann machine. We prepared three sets of different pictures, aerial, face, and forest. To reduce the huge computational time of the learning process, we used the NMF, which enabled us to handle relatively large patch sizes up to $L=32$. The results are stable against a change in system size if the linear size $L$ is larger than or equal to $L=16$. The refined mean-field method, the BA, was also employed to check the validity of the NMF, and we found that the NMF results are reliable for the interactions among 
the sets of pictures we studied. This conclusion was reinforced by using the Monte Carlo method for small sizes and by examining other dithering methods.

As individual characteristics of each set of pictures, we found that the NN and NNN interactions strongly depend on the set of pictures. Both negative and positive values can appear for these $\mathrm{NN}$ and NNN interactions. Meanwhile, as universal aspects, we observed that the inferred interactions are essentially short-range. For a distance longer than $r \geq 2$, the interactions basically are positive and decay rapidly among all the sets of pictures. The characteristic length scale is commonly about $\xi \approx 4$. Simple periodic behaviors are also observed in all the cases. Summarizing these properties, we proposed a model prior distribution with six parameters at most. It will be interesting future work to examine the performance of this model distribution in image processing tasks, such as image restoration.

As an additional topic, we also examined the concepts of frustration and criticality. Frustration is present for the interactions inferred from the face pictures, but absent for the other two sets of pictures. In all the cases, the criticality is not observed in the Boltzmann machine after learning, although our binarized images show a clear power law in the Fourier amplitude plotted against the absolute value of the Fourier frequency. We speculate that this is a weak point of the Boltzmann machine, which employs only up to the second order statistics; the criticality in natural images would be sustained by higher order statistics than the second.

The relation to simple-cell receptive fields was also examined. Our results imply that it can be better to tune the distribution of the sparse coefficients according to the target images. This distribution should have a nontrivial functional form different from Gaussian. Constructing the functional form and determining the hyper parameters also constitute interesting future work.

\section{Acknowledgments}

T. O. is grateful to Y. Kabashima, M. Kikuchi, and K. Tokita for fruitful discussions. This work was supported by Grant-in-Aid for JSPS Fellows (No. 2011) (TO), KAKENHI No. 26870185, 25120013 (TO) and No. 15K00330 (MY). 


\section{References}

1) Geman S and Geman D 1984 Stochastic relaxation, Gibbs distributions and the Bayesian restoration of images IEEE Trans. PAMI 6 721-741

2) Ruderman D L and Bialek W 1994 Statistics of natural images: Scaling in the woods Phys. Rev. Lett. 73 814-817

3) Ruderman D L 1994 The statistics of natural images Network: Computation in Neural Systems $5517-548$

4) Olshausen B A and Field D J 1996 Emergence of simple-cell receptive field properties by leaning a sparse code for natural images Nature 381 607-609

5) E. P. Simoncelli and B. A. Olshausen 2001 Natural Image Statistics and Neural Representation Annu. Rev. Neurosci. 24 1193-1216

6) Tanaka K 2002 Statistical-mechanical approach to image processing (Topical review) J. Phys. A: Math. Gen. 35 81-150

7) Hyvärinen A, Hurri J, and Hoyer P. O. 2009 Natural Image Statistics -A probabilistic approach to early computational vision (Springer)

8) G.E. Hinton and R.R. Salakhutdinov, Reducing the Dimensionality of Data with Neural Networks, Science, 2006, 313504 - 507.

9) G.E. Hinton, S. Osindero, and Y. Teh, 2006 A fast learning algorithm for deep belief nets, Neural Computation, 18

10) Y. Bengio, Learning deep architectures for AI, 2009 Foundations and Trends in Machine Learning Vol. 2 (1) 1-127.

11) Ackley D H, Hinton G E and Sejnowski T J 1985 A Learning Algorithm for Boltzmann Machines Cognitive Science 9 147-169

12) Krähenbühl P and Koltun V 2011 Efficient Inference in Fully Connected CRFs with Gaussian Edge Potentials In Proc. NIPS 109-117

13) Stephens G J, Mora T, Tkačik G and Bialek W 2013 Statistical Thermodynamics of Natural Images Phys. Rev. Lett. 110018701

14) Peterson C and Anderson J R 1987 A Mean Field Theory Learning Algorithm for Neural Networks Complex Systems 1 995-1019

15) Hinton G E 1989 Deterministic Boltzmann Learning Performs Steepest Descent in WeightSpace Neural Computation 1 143-150 
16) Kappen H J and Rodrìguez F B 1997 Efficient learning in Boltzmann Machines using linear response theory Neural Computation $\mathbf{1 0} 1137$

17) Yasuda M and Tanaka K 2012 Approximate Learning Algorithm in Boltzmann Machines, Neural Computation 21(11) 3130-3178

18) Ricci-Tersenghi F 2012 The Bethe approximation for solving the inverse Ising problem: a comparison with other inference methods J. Stat. Mech. P08015

19) Opper M and Saad D 2001 Advanced mean field methods: theory and practice (The MIT press)

20) Mezard M and Montanari A 2009 Information, Physics and Computation (Oxford University Press)

21) Databese of Geospatial Information Authority of Japan: http://www.gsi.go.jp/tizu-kutyu.html

22) Color FERET: http://www.nist.gov/itl/iad/ig/colorferet.cfm

23) SUN Database: http://groups.csail.mit.edu/vision/SUN/

24) ImageMagick: http://www.imagemagick.org/script/index.php

25) Quantization: http://www.imagemagick.org/script/quantize.php

26) Riemersma: http://www.compuphase.com/riemer.htm

27) Baxter R J 2007 Exactly Solved Models in Statistical Physics (Dover Publications)

28) J. P. Jones and L. A. Palmer: An evaluation of the two-dimensional Gabor filter model of simple receptive fields in cat striate cortex, Journal of Neurophysiology, 198758 1233-1258

29) sparsenet: http://redwood.berkeley.edu/bruno/sparsenet/ 